\title{
Motion planning and numerical simulation of material deposition at corners in extrusion additive manufacturing
}

\author{
Comminal, Raphaël; Serdeczny, Marcin P.; Pedersen, David B.; Spangenberg, Jon
}

Published in:

Additive Manufacturing

Link to article, DOI:

10.1016/j.addma.2019.06.005

Publication date:

2019

Document Version

Peer reviewed version

Link back to DTU Orbit

Citation (APA):

Comminal, R., Serdeczny, M. P., Pedersen, D. B., \& Spangenberg, J. (2019). Motion planning and numerical simulation of material deposition at corners in extrusion additive manufacturing. Additive Manufacturing, 29, [100753]. https://doi.org/10.1016/j.addma.2019.06.005

\section{General rights}

Copyright and moral rights for the publications made accessible in the public portal are retained by the authors and/or other copyright owners and it is a condition of accessing publications that users recognise and abide by the legal requirements associated with these rights.

- Users may download and print one copy of any publication from the public portal for the purpose of private study or research.

- You may not further distribute the material or use it for any profit-making activity or commercial gain

- You may freely distribute the URL identifying the publication in the public portal 


\title{
Motion planning and numerical simulation of material deposition at corners in extrusion additive manufacturing
}

\author{
Raphaël Comminal*, Marcin P. Serdeczny, David B. Pedersen, Jon Spangenberg
}

Department of Mechanical Engineering, Technical University of Denmark, Kgs. Lyngby, Denmark

*Corresponding author: $\underline{\text { rcom@mek.dtu.dk }}$

Keywords: Material extrusion additive manufacturing; Deposition flow; Toolpath motion planning; Corner swelling; Corner rounding.

\section{Abstract}

The material deposition along a toolpath with a sharp corner is simulated with a computational fluid dynamics model. We investigate the effects of smoothing the toolpath and material over-extrusion on the corner rounding and the corner swelling, for $90^{\circ}$ and $30^{\circ}$ turns. The toolpath motion is controlled with trapezoidal velocity profiles constrained by a maximal acceleration. The toolpath smoothing of the corner is parametrized by a blending acceleration factor. Analytical solutions for the deviation of the smoothed toolpath from the trajectory of the sharp corner, as well as the additional printing time required by the deceleration and acceleration phases in the vicinity of the turn are provided. Moreover, several scenarios with different blending acceleration factors are simulated, for the cases of a constant extrusion rate and an extrusion rate proportional to the printing head speed. The constant extrusion rate causes material over-extrusion during the deceleration and acceleration phases of the printing head. However, the toolpath smoothing reduces the corner swelling. The amount of underfill and overfill at the inside and outside of the corner are quantified, as compared to an ideal case where the printing head would instantaneously change its speed direction at the corner. The numerical results show that there is an optimal amount of toolpath smoothing where the over-extrusion compensates for the corner rounding; hence improving the quality of the corner. A uniform road width is obtained with the proportional extrusion rate.

\section{Introduction}

Material extrusion Additive Manufacturing (AM), also known as Fused Deposition Modeling (FDM) and Fused Filament Fabrication (FFF), has become one of the most popular 3D printing techniques for thermoplastics. In this process, molten material is extruded from a movable printing head and deposited along a prescribed road. During extrusion, feedstock material is continuously supplied to the printing head in the form of a solid filament, driven by a pinch roller mechanism. The printing head consists of a liquefier that melts the filament and an extrusion nozzle which deposits a molten strand. Besides providing feedstock, the solid filament also acts as a piston which pushes the molten plastic through the extrusion nozzle. After deposition, the extruded material cools down and solidifies onto the substrate, forming an object layer by layer. Most of the important properties of the finished parts, including the dimensional accuracy, surface roughness, internal porosity and mechanical strength, depend on the deposition path as well as the shape of the deposited strand [1-3].

Before fabrication, the CAD model of the part is processed by a slicer software that generates the G-code containing all the machine instructions. Most slicer software breaks down curved trajectories into linear segments. During the fabrication, the G-code is interpreted by the firmware that controls the different actuators of the machine. The G-code is a machine-neutral file that prescribes the displacements and the speed of the printing head, the filament feeding rate, the extruder temperature, etc. The G-code may also specify some configuration parameters of the machine, such as the maximum acceleration of the axes. However, the acceleration planning is managed by the firmware of the 3D printer.

Go et al. [4] identified the force capacity of the feeding system and the heat capacity of the liquefier as two of the main limitations (together with the gantry dynamics) for faster build rate. Subsequently, Go and Hart [5] achieved remarkable enhancement of the build rate of a desktop 3D printing, by improving the robustness of the feeding system, the liquefier, and the gantry. Weiss et al. [6] developed a closed-loop proportional-integral controller of the gantry that improves the acceleration and positioning accuracy of an open-source 3D printer. Nevertheless, according to Go and Hart [5], faster material extrusion AM will also require improved toolpath planning algorithms, and coupled high-speed motion and extrusion dynamics [4].

Bouhal et al. [7] developed a look-ahead toolpath planning algorithm that decelerates the printing head when approaching corners, in order to reduce positioning errors due to the saturation of the motors during turns where large accelerations are required. Han et al. [8] identified different reasons for overfill and underfill material deposition (among which is the decrease of the printing head speed at turns), and proposed a deposition planning algorithm that improved the layer evenness. In a subsequent study, Han and Jafari [9] proposed a coordination control of the deposition rate and the positioning of the printing head by taking into account their phase lag. The implementation of this control approach is challenging because the time constants in the transfer functions of the 
different subsystem (i.e. the filament drivers, the liquefier-extruder and the strand forming) are generally unknown. Ertay et al. [10] proposed a proportional control of the extrusion rate and the printing head speed, synchronized with the power supply of the heaters. In addition, Ertay et al. [10] implemented a global smoothing algorithm of the toolpath with rational B-splines, which was shown to improve the regularity of the material deposition along roads with sharp curvature. Giberti et al. [11,12] proposed a path planning algorithm that maintains a constant tangential velocity during the turns, by smoothing the corners with quadratic Bézier curves.

Greeff and Schilling [13] developed a closed-loop control system of the filament feeding system, using a microscope video camera to monitor the volumetric flow rate of the filament. Their measurements showed that the amount of slippage-which depends on the pressure drop inside the extruder-is influenced by the extrusion temperature and feed rate. Moreover, they found that the slippage varies dynamically, and therefore cannot be fully corrected statically. Jiang and Gu [14] described an extrusion instability mechanism occurring when the required force to extrude the material exceeds the maximum torque that can be provided by the motors of the filament drivers, leading to fluctuations in the pressure drop and extrusion rate. Mackey et al. [15] showed a correlation between the extrusion temperature and the maximum feed velocity before failure. They found that the maximum feed rate depends on the thermal and rheological properties of the molten plastic.

Peng et al. [16] reported experimental measurements of the shear rate and temperature history inside the liquefier, highlighting the non-Newtonian and non-isothermal characteristics of the flow. Pollard et al. [17] measured via infra-red emissions the transient temperature response of the extruded material when the filament feed rate was subjected to step variations. Numerical and analytical thermo-fluid models of steady-state liquefiers have been presented in $[18,19]$. These models include a shear thinning temperature dependent viscosity function and the latent heat of fusion of the thermoplastic. Experimental investigations of Phan et al. [20] have shown that the relation between the pressure drop and the extrusion rate is non-monotonic. A simplified dynamical model of the liquefier-extruder system was proposed by Bellini et al. [21]. They derived an analytical transfer function of the liquefier, and showed that the step response of the extrusion rate is subjected to both time delay and amplitude errors. Their model demonstrates that time delays in the extrusion rate come from the torque limitation of the motors and the strong temperature dependency of the molten plastic viscosity, while the steady amplitude errors are due to slippage of the drive rollers on the filament.

The deposition flow and the strand forming of extruded material was modeled with Computational Fluid Dynamics (CFD) simulations in [22-28]. Du et al. [22] and Xia et al. [23,24] focused on predicting the thermal field, to determine the cooling rate and the reheating effect due to successive layer deposition. Comminal et al. [25] used an isothermal model and investigated the influence of the printing speed and the layer thickness on the shape of the deposited strand. The predictions of the CFD model were validated by experiments in [26]. In a further work, Serdeczny et al. [27] simulated the successive deposition of parallel strands, in order to predict the porosity as well as the inter-layer and intra-layer bonding areas inside the part. Simulations of strand forming at the corners have been presented in a preliminary study of Comminal et al. [28].

Understanding and predicting the occurrence of overfill and underfill at corners have important applications in the development of novel toolpath generation algorithms to improve fabrication accuracy [29,30]. In this paper, we use an isothermal CFD model to simulate the material deposition along toolpaths with a sharp corner. The numerical model is used to determine the amount of overfill and underfill, at the inside and outside of the turn, for $90^{\circ}$ and $30^{\circ}$ corners. Smoothed printing head motions with different trapezoidal velocity profiles are simulated, for constant and proportional extrusion rates. The purpose of the simulations is to identify an optimal way to travel along the corners. The rest of the paper is organized as follows. Section 2 discusses experimental observations of deposition defects at the corners. Section 3 introduces a parametric motion planning of the printing head with trapezoidal velocity profiles that are used in the numerical simulations. Section 4 presents the computational model and the numerical methods, while the results are discussed in Section 5.

\section{Experimental observations}

Parts manufactured by material extrusion AM often have undesirable features that negatively affect the quality of the corners. Common corner defects include corner rounding, corner swelling and corner ringing, which are very well known in the fused filament fabrication community, as reflected for instance in [31]. Those defects can be more or less pronounced, depending on the configuration and calibration of the 3D printer.

Corner rounding happens because of local smoothing of the sharp corners, when the printing head negotiates turns at high speed. The amount of corner rounding depends on the printing speed and firmware configuration. Corner swelling is due to material over-extrusion forming a bulge at the corner. The over-extrusion comes from the fact the liquefier continues to extrude material while the speed of the printing head is reduced when approaching the corner. Over-extrusion can also occur despite reducing the feeding speed, because the compressed filament continues to apply a pressure on the molten plastic as it is releasing stored elastic energy. Corner ringing is an instability at the edges of corners, which is a result of vibrations in the positioning system, due to sudden variations of the traction forces. Corner ringing is more pronounced at large accelerations. However, the ringing instability can be reduced by improving rigidity of the mechanical system. 
To illustrate these phenomena, we printed strands with $90^{\circ}$ corners on a BQ Hephestos 2 desktop 3D-printer. The experiments were carried out using PLA material and the extrusion temperature of $200^{\circ} \mathrm{C}$. Some of the printed samples with distinct defects are shown in Fig. 1. The $90^{\circ}$ corners were printed with different layer heights $h$ and printing speeds $V$, but with the same ratio of feeding speed $V_{f}$ over printing speed (the ratio was set to $V_{f} / V=$ $\left(D / D_{f}\right)^{2} \approx 0.052$, where $D=0.4 \mathrm{~mm}$ is the nozzle diameter and $D_{f}=1.75 \mathrm{~mm}$ is the mean filament diameter). However, in our experiments, the defects primarily correlated with the position of the printing head in the build plane rather than the layer height or the printing speed.

(a)

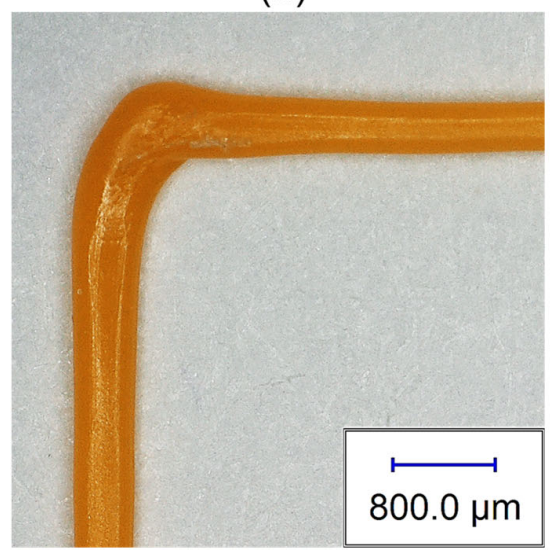

(b)

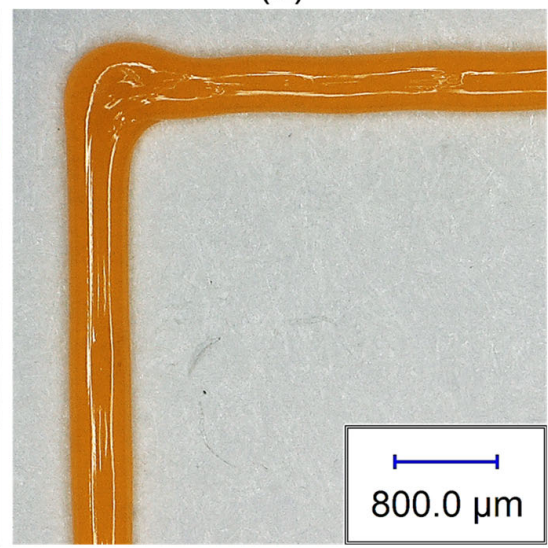

(c)

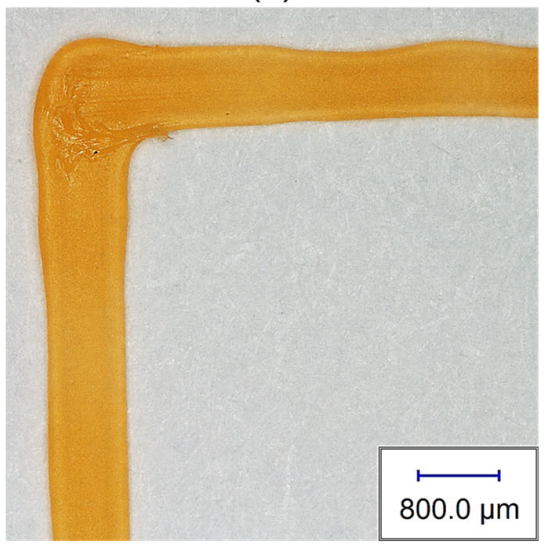

Fig. 1. Undesired features at the corner: (a) corner rounding $(h=0.4 \mathrm{~mm} ; V=200 \mathrm{~mm} / \mathrm{s})$, (b) corner swelling $(h=$ $0.4 \mathrm{~mm} ; V=40 \mathrm{~mm} / \mathrm{s})$, (c) corner ringing $(h=0.32 \mathrm{~mm} ; V=80 \mathrm{~mm} / \mathrm{s})$. The printing head turns from the vertical segment ( $Y$ axis) to the horizontal segment ( $X$ axis).

The BQ Hephestos 2 desktop 3D printer has an aluminum frame with a Cartesian coordinate system, where the printing head navigates on the $X$ and $Z$ axes while the build plate moves along the $Y$ axis. The $X$ and $Z$ axes slide on recirculating ball bearings guides with flat runners; the $Y$ axis runs on hard chromed bars with linear ball bearings. The motion along each axis is powered by stepper motors and transmitted through groove rubber belts. The 3Dprinter was operated with the vendor-provided firmware version 2.5.1, using the recommended configuration for BQ Hephestos 2. The firmware controls the motion with a combination of trapezoidal velocity profiles with a maximum acceleration of $1000 \mathrm{~mm} / \mathrm{s}^{2}$ and velocity step functions with a jerk value of $20 \mathrm{~mm} / \mathrm{s}$. Here, the jerk corresponds to the maximum velocity variation ${ }^{1}$ that is assumed to occur instantaneously by the firmware. Thus, the firmware only plans acceleration phases with trapezoidal velocity functions for the velocity variations above the jerk value. Velocity variations below the jerk value are scheduled with velocity step functions. In practice, the actuators do not have an infinite acceleration, but the step function commands the motors to accelerate as fast as possible. However, the increase of torque during acceleration generates lateral forces on the axes, and elastic stretch of the transmission rubber belt, which result in vibrations of the frame, and oscillations of the printing head position. The vibrations and oscillations are damped by the internal friction of the system and the energy dissipation of the rubber belt. Therefore, the jerk value can be seen as an adjustable parameter that influences the proneness to corner ringing and corning swelling.

In general, the shape of the deposited strand along the corner is affected by a combination of the rounding, swelling and ringing phenomena. One of the advantages of computational simulations is that they can isolate the effects of those different phenomena. Our current numerical simulations do not include the ringing instability, as a perfectly rigid positioning system is assumed. Nevertheless, the simulations can be used to investigate the influence of the toolpath planning on the corner rounding and to quantify the amount of corner swelling caused by the constant extrusion rate during a turn.

\section{Corner printing strategy}

\subsection{Printing head motion}

This subsection introduced a parametric model describing how the printing head can smoothly travel along sharp corners using trapezoidal velocity profiles. We assume that the printing head strives to move along linear

\footnotetext{
${ }^{1}$ Not to be confused with the time derivative of the acceleration, which is also referred to as the "jerk" in the field of CNC machining.
} 
segments with the target speed prescribed in the G-code, whenever possible. However, the printing head must decrease its cruising speed in the vicinity of the corner, in order to correctly negotiate the turn. An instantaneous change of the printing direction while travelling at the cruising speed would require an infinite acceleration. That motion is unrealistic; however, it would be an ideal motion, in the sense that it follows exactly the prescribed toolpath, while traveling at the expected speed all along the turn. In the following, we refer to this motion as the ideal turn, as it is an unachievable motion that we would like to approach.

The only physical way (without infinite acceleration) to follow the exact toolpath of the sharp corner is to stop the printing head at the tip of the corner. Thus, the printing head should anticipate the turn by starting decelerating before reaching the end of the first segment. Then, the printing head would start accelerating along the direction of the second segment just after its stop at the corner, until the cruising speed is reached. We refer to this trajectory as the stop-at-turn motion. The stop-at-turn trajectory follows exactly the prescribed toolpath. However, because of the deceleration and acceleration phases, the stop-at-turn trajectory takes a longer travelling time than the ideal turn. The additional time spent to decelerate and accelerate the printing head decreases the average printing speed along the segments. The total increase of the printing time can become substantial in the case of toolpaths comprising many short linear segments (which is the typical way how curved trajectory are represented).

Alternatively, the printing head can be accelerated and decelerated along both segments at the same time, in order to minimize the printing time. In this case, however, the trajectory of the printing head does not follow the exact toolpath and describes a smoothed corner rather than a sharp corner. We refer to this trajectory as the blended acceleration motion. The blended acceleration trajectory minimizes the printing time at the expense of rounding the corner.

For the sake of reaching a trade-off between quality and manufacturing time, one may wish to have a compromise in between the two limiting case of the stop-at-turn trajectory and the blended acceleration trajectory. Thus, we also consider the case of the partially blended acceleration trajectory, where the printing head decelerates along the linear segment as it approaches to the corner, before negotiating the turn with blended acceleration, at a lower speed. In that way, the partially blended acceleration trajectory has a smaller deviation from the prescribed sharp toolpath as compared to the fully blended acceleration turn, while printing faster than for the stop-at-turn trajectory. The partially blended acceleration profile is parametrized by the blending acceleration factor $\kappa$, with a value between 0 and 1 , where $\kappa=1$ for the fully blended acceleration trajectory and $\kappa=0$ in the stop-at-turn trajectory.

For the sake of simplicity, we used a parametric model with trapezoidal velocity profiles along the axes, with step function accelerations bounded by the maximum acceleration $A_{\max }$. The sharp corner is defined by two successive linear segments $\overrightarrow{S_{1}}$ and $\overrightarrow{S_{2}}$ of the toolpath. Each segment $\overrightarrow{S_{l}}$, where $i=1,2$, can be characterized by its length $L_{i}$, its polar angle to the $X$ axis $\alpha_{i}$, and its target cruising speed $V_{i}$. Fig. 2 represents an arbitrary corner $\widehat{\mathrm{AOF}}$, where the segment lines $\mathrm{AO}$ and OF correspond to $\overrightarrow{S_{1}}$ and $\overrightarrow{S_{2}}$. The printing head trajectories described above generally require velocity profiles with 5 phases:

1. the cruising phase at constant speed $V_{1}$, along the segment line $\mathrm{AB}$,

2. the deceleration phase while approaching the corner, along the segment line BC (except when $\kappa=1$ ),

3. the blended acceleration phase in the vicinity of the corner, along the curve CD (except when $\kappa=0$ ),

4. the acceleration phase to move away from the corner, along the segment line DE (except when $\kappa=1$ ),

5. the cruising phase at constant speed $V_{2}$, along the segment line EF.

When $\kappa=0$, the points $\mathrm{C}$ and $\mathrm{D}$ coincide with the corner point $\mathrm{O}$. When $\kappa=1$, the points $\mathrm{C}$ and $\mathrm{D}$ coincide with the points $B$ and E, respectively. Examples of acceleration and velocity profiles for $90^{\circ}$ turns where the two segments are aligned with the $X$ and $Y$ axes are show in Fig. 3, for $\kappa=0.5$ (partially blended accelerations) and $\kappa=1$ (fully blended accelerations). 
1 3 4 5
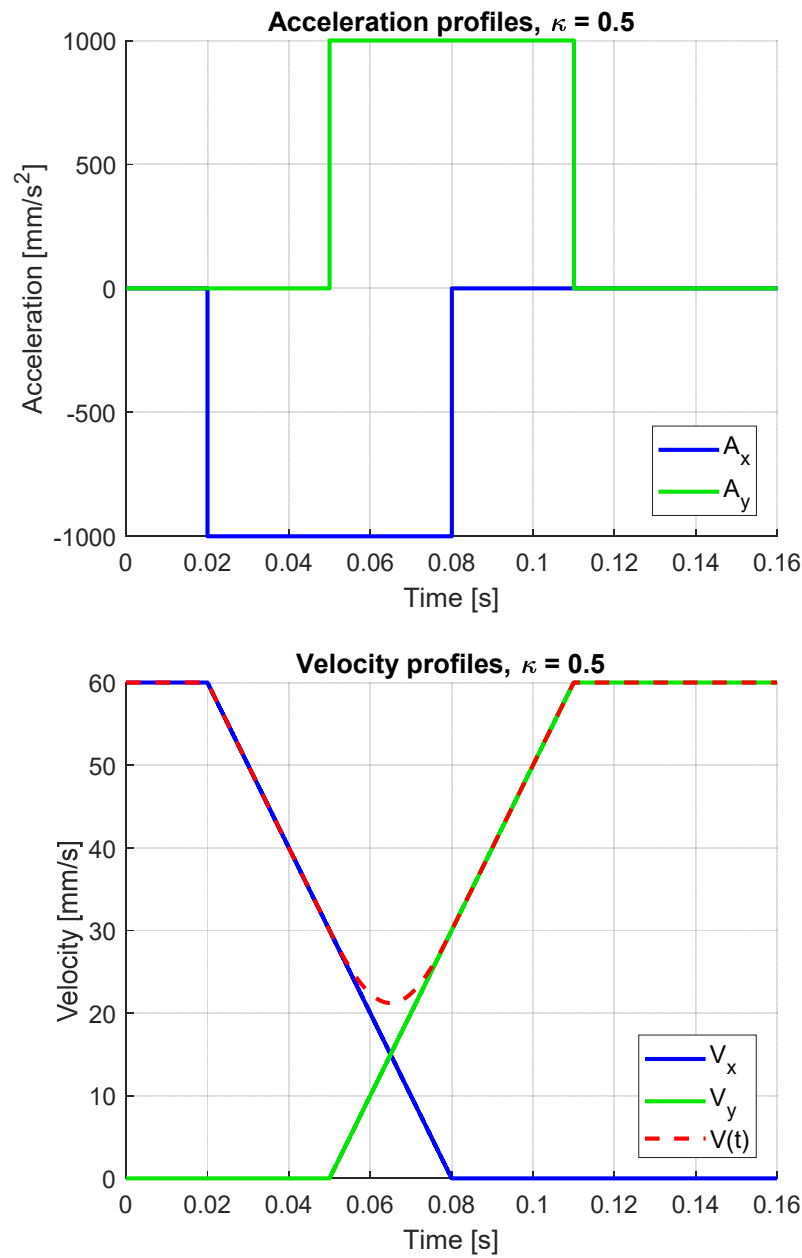

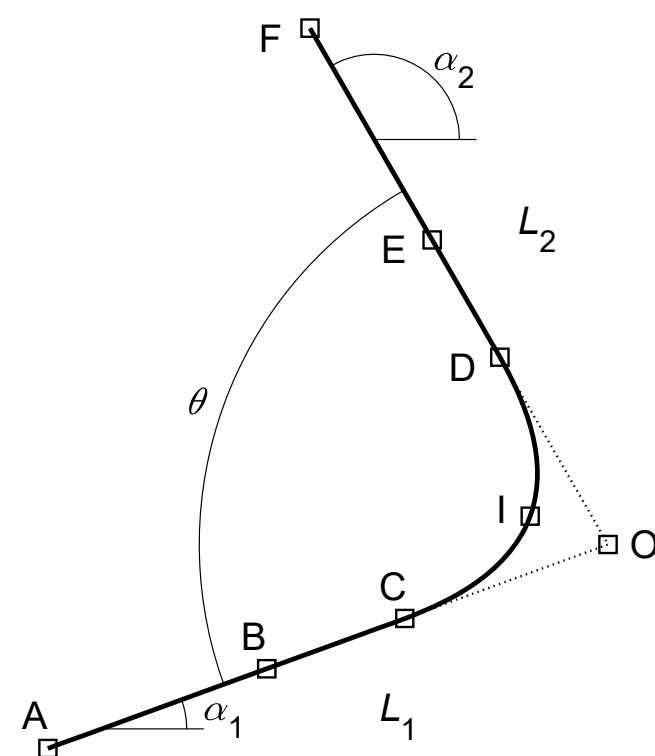

Fig. 2. Smoothed trajectory along an arbitrary turn.
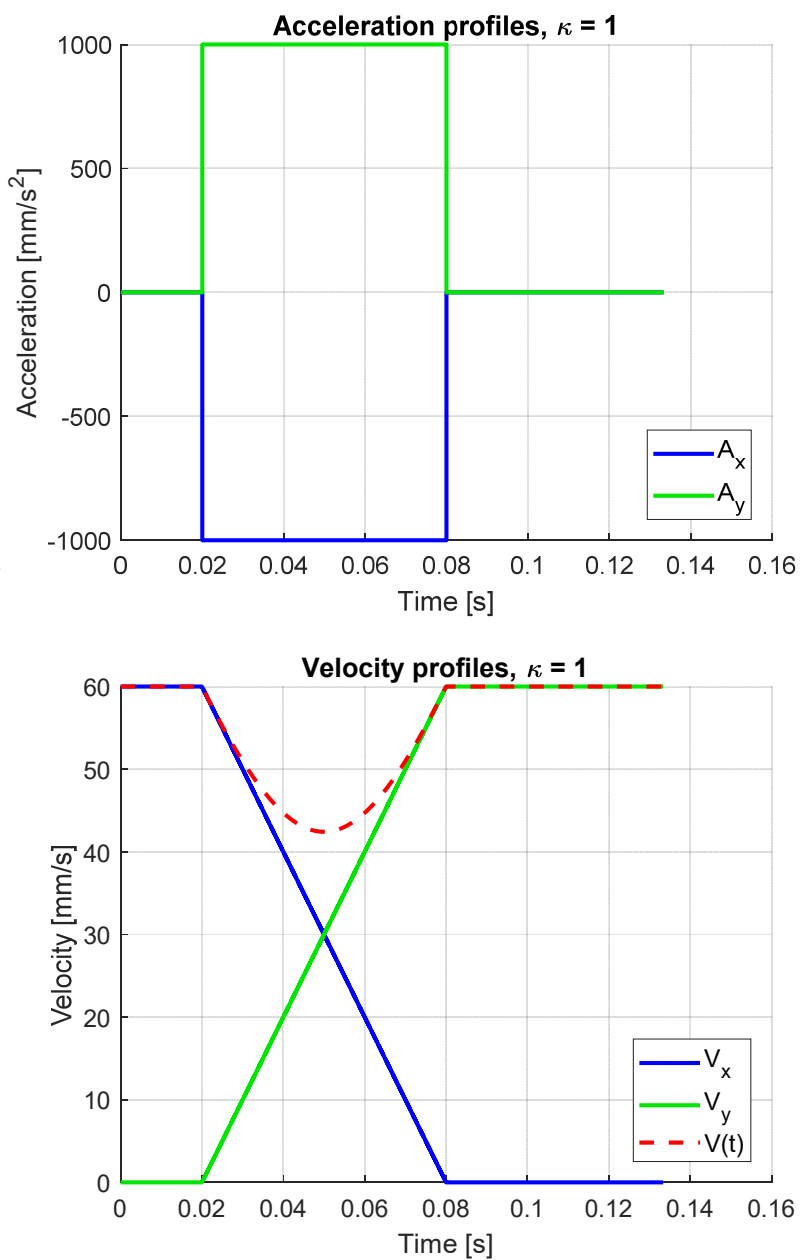

Fig. 3. Examples of acceleration and velocity profiles for $90^{\circ}$ turns where $\kappa=0.5$ and $\kappa=1$. $\left(V_{1}=V_{2}=60 \mathrm{~mm} / \mathrm{s}\right.$; $A_{\max }=1000 \mathrm{~mm} / \mathrm{s}$; the segments are aligned with $X$ and $Y$ axes.) 
The instantaneous velocity of the printing head is:

$$
V(t)=\sqrt{V_{x}(t)^{2}+V_{y}(t)^{2}},
$$

2 where $V_{x}(t)$ and $V_{y}(t)$ are the velocity components along the $X$ and $Y$ axes, respectively. The projections of the

3 cruising speeds $V_{1}$ and $V_{2}$ along the $X$ and $Y$ axes are:

$$
\begin{aligned}
& V_{x_{S 1}}=V_{1} \cos \left(\alpha_{1}\right), \\
& V_{y_{S 1}}=V_{1} \sin \left(\alpha_{1}\right), \\
& V_{x_{S 2}}=V_{2} \cos \left(\alpha_{2}\right), \\
& V_{y_{S 2}}=V_{2} \sin \left(\alpha_{2}\right) .
\end{aligned}
$$

Let $t_{\mathrm{B}}, t_{\mathrm{C}}, t_{\mathrm{D}}$ and $t_{\mathrm{E}}$ be the time when the printing head reaches the position $\mathrm{B}, \mathrm{C}, \mathrm{D}$ and $\mathrm{E}$, respectively. During the different phases of the turn, the acceleration step functions and their corresponding trapezoidal velocity profiles are:

- $\quad$ for $t \leq t_{\mathrm{B}}$ : (cruising phase along $\mathrm{AB}$ )

$$
\begin{gathered}
A_{x}(t)=0 \\
A_{y}(t)=0 \\
V_{x}(t)=V_{x_{S 1}} \\
V_{y}(t)=V_{y_{S 1}}
\end{gathered}
$$

- for $t_{\mathrm{B}}<t \leq t_{\mathrm{C}}$ : (deceleration phase along $\mathrm{BC}$ )

$$
\begin{gathered}
A_{x}(t)=\left(\kappa_{1}-1\right) V_{x_{S 1}} / \Delta t_{\mathrm{BC}} \\
A_{y}(t)=\left(\kappa_{1}-1\right) V_{y_{S 1}} / \Delta t_{\mathrm{BC}} \\
V_{x}(t)=(1-\xi(t)) V_{x_{S 1}}+\xi(t) \kappa_{1} V_{x_{S 1}} \\
V_{y}(t)=(1-\xi(t)) V_{y_{S 1}}+\xi(t) \kappa_{1} V_{y_{S 1}} \\
\xi(t)=\left(t-t_{\mathrm{B}}\right) /\left(t_{\mathrm{C}}-t_{\mathrm{B}}\right)
\end{gathered}
$$

- for $t_{\mathrm{C}}<t \leq t_{\mathrm{D}}$ : (blended acceleration phase along $\mathrm{CD}$ )

$$
\begin{gathered}
A_{x}(t)=\left(\kappa_{2} V_{x_{S 2}}-\kappa_{1} V_{x_{S 1}}\right) / \Delta t_{\mathrm{CD}} \\
A_{y}(t)=\left(\kappa_{2} V_{y_{S 2}}-\kappa_{1} V_{y_{S 1}}\right) / \Delta t_{\mathrm{CD}} \\
V_{x}(t)=(1-\xi(t)) \kappa_{1} V_{x_{S 1}}+\xi(t) \kappa_{2} V_{x_{S 2}} \\
V_{y}(t)=(1-\xi(t)) \kappa_{1} V_{y_{S 1}}+\xi(t) \kappa_{2} V_{y_{S 2}} \\
\xi(t)=\left(t-t_{\mathrm{C}}\right) /\left(t_{\mathrm{D}}-t_{\mathrm{C}}\right)
\end{gathered}
$$

- for $t_{\mathrm{D}}<t \leq t_{\mathrm{E}}$ : (acceleration phase along DE)

$$
\begin{gathered}
A_{x}(t)=\left(1-\kappa_{2}\right) V_{x_{S 2}} / \Delta t_{\mathrm{DE}} \\
A_{y}(t)=\left(1-\kappa_{2}\right) V_{y_{S 2}} / \Delta t_{\mathrm{DE}} \\
V_{x}(t)=(1-\xi(t)) \kappa_{2} V_{x_{S 2}}+\xi(t) V_{x_{S 2}} \\
V_{y}(t)=(1-\xi(t)) \kappa_{2} V_{y_{S 2}}+\xi(t) V_{y_{S 2}} \\
\xi(t)=\left(t-t_{\mathrm{D}}\right) /\left(t_{\mathrm{E}}-t_{\mathrm{D}}\right)
\end{gathered}
$$

- for $t_{\mathrm{E}}<t$ : (cruising phase along $\mathrm{EF}$ )

$$
\begin{gathered}
A_{x}(t)=0 \\
A_{y}(t)=0 \\
V_{x}(t)=V_{x_{S 2}} \\
V_{y}(t)=V_{y_{S 2}}
\end{gathered}
$$

where $\xi(t)$ is an auxiliary function varying linearly from 0 to 1 during each acceleration/deceleration phase (i.e. it is a normalized time). In order to obtain a symmetric trajectory with respect to the bisector of the angle $\overline{\mathrm{AOF}}$, coefficients of speed reduction during the deceleration and acceleration phases along $\mathrm{BC}$ and $\mathrm{DE}$ are chosen as:

$$
\begin{aligned}
& \kappa_{1}=\kappa \min \left(1, V_{2} / V_{1}\right), \\
& \kappa_{2}=\kappa \min \left(1, V_{1} / V_{2}\right) .
\end{aligned}
$$

The durations of the acceleration and deceleration phases depend on the largest of the velocity variations among the $X$ and $Y$ axes. For instance, $\Delta t_{\mathrm{BC}}=t_{\mathrm{C}}-t_{\mathrm{B}}$ is either determined from Eq. (4.1) or (4.2), depending on the largest value among $\left|\left(\kappa_{1}-1\right) V_{x_{S 1}}\right|$ and $\left|\left(\kappa_{1}-1\right) V_{y_{S 1}}\right|$. Similarly, the durations $\Delta t_{\mathrm{CD}}=t_{\mathrm{D}}-t_{\mathrm{C}}$ and $\Delta t_{\mathrm{DE}}=t_{\mathrm{E}}-t_{\mathrm{D}}$ are either determined from Eq. (5.1) or (5.2), and Eq. (6.1) or (6.2), respectively. Thus, the durations of the acceleration and deceleration phases correspond to: (details for deriving the final form of Eq. (9.2) are provided in Appendix A)

$$
\begin{gathered}
\Delta t_{\mathrm{BC}}=\frac{1}{A_{\max }} \max \left(\left|\left(\kappa_{1}-1\right) V_{x_{S 1}}\right|,\left|\left(\kappa_{1}-1\right) V_{y_{S 1}}\right|\right)=\frac{V_{1}-\kappa \min \left(V_{1}, V_{2}\right)}{A_{\max } f\left(\alpha_{1}\right)}, \\
\Delta t_{\mathrm{CD}}=\frac{1}{A_{\max }} \max \left(\left|\kappa_{2} V_{x_{S 2}}-\kappa_{1} V_{x_{S 1}}\right|,\left|\kappa_{2} V_{y_{S 2}}-\kappa_{1} V_{y_{S 1}}\right|\right) \\
=\frac{\kappa \min \left(V_{1}, V_{2}\right)}{A_{\max }} \max \left(\left|\cos \left(\alpha_{2}\right)-\cos \left(\alpha_{1}\right)\right|,\left|\sin \left(\alpha_{2}\right)-\sin \left(\alpha_{1}\right)\right|\right) \\
=\frac{2 \kappa \min \left(V_{1}, V_{2}\right)}{A_{\max } f\left(\frac{\alpha_{2}+\alpha_{1}}{2}\right)} \cos \left(\frac{\theta}{2}\right),
\end{gathered}
$$




$$
\Delta t_{\mathrm{DE}}=\frac{1}{A_{\max }} \max \left(\left|\left(1-\kappa_{2}\right) V_{x_{S 2}}\right|,\left|\left(1-\kappa_{2}\right) V_{y_{S 2}}\right|\right)=\frac{V_{2}-\kappa \min \left(V_{1}, V_{2}\right)}{A_{\max } f\left(\alpha_{2}\right)},
$$

where $f(\alpha)$ is the real function defined as:

$$
f(\alpha)=\frac{1}{\max (|\cos (\alpha)|,|\sin (\alpha)|)}, \forall \alpha \in \mathbb{R},
$$

and $\theta$ is the smallest angle (i.e. in the interval [0, $\pi]$ ) formed by the two segments (see Fig. 2):

$$
\theta=\min \left(\alpha_{2}-\alpha_{1}-\pi \bmod 2 \pi, 2 \pi-\left(\alpha_{2}-\alpha_{1}-\pi \bmod 2 \pi\right)\right) \text {. }
$$

The function $f(\alpha)$ varies between 1, for angles $\bmod \left(\alpha, 90^{\circ}\right)=0^{\circ}$ (i.e. for segments parallel to either the $X$ or $Y$ axes), and $\sqrt{2}$, for angles $\bmod \left(\alpha, 90^{\circ}\right)=45^{\circ}$ (i.e. for segments in the diagonal directions of the $X$ and $Y$ axes). The function $f(\alpha)$ reflects the fact that the printing head can reach a larger acceleration when moving along diagonal directions, because of the combined accelerations along both the $X$ and $Y$ axes (i.e. the combined maximum acceleration along diagonal directions is $\sqrt{2} A_{\max }$ ).

The distance travelled during the deceleration phase along $\mathrm{BC}$ and the acceleration phase along $\mathrm{DE}$ are respectively:

$$
\begin{aligned}
& L_{\mathrm{BC}}=\overline{V_{\mathrm{BC}}} \Delta t_{\mathrm{BC}}=\frac{1+\kappa_{1}}{2} V_{1} \Delta t_{\mathrm{BC}}, \\
& L_{\mathrm{DE}}=\overline{V_{\mathrm{DE}}} \Delta t_{\mathrm{DE}}=\frac{1+\kappa_{2}}{2} V_{2} \Delta t_{\mathrm{DE}}
\end{aligned}
$$

where $\overline{V_{\mathrm{BC}}}$ and $\overline{V_{\mathrm{DE}}}$ are average velocities along segments $\mathrm{BC}$ and $\mathrm{DE}$, respectively. The distance between the points $\mathrm{C}$ and $\mathrm{D}$ is (see derivation in Appendix B):

$$
L_{\mathrm{CD}}=\kappa \min \left(V_{1}, V_{2}\right) \sin \left(\frac{\theta}{2}\right) \Delta t_{\mathrm{CD}}
$$

Moreover, the points $\mathrm{C}$ and $\mathrm{D}$ are equidistant to the corner point $\mathrm{O}$ and the length of the segments $\mathrm{CO}$ and $\mathrm{OD}$ is (see derivation in Appendix B):

$$
L_{\mathrm{CO}}=L_{\mathrm{OD}}=\frac{\left[\kappa \min \left(V_{1}, V_{2}\right)\right]^{2}}{A_{\max } f\left(\frac{\alpha_{2}+\alpha_{1}}{2}\right)} \cos \left(\frac{\theta}{2}\right) .
$$

The distances $L_{\mathrm{BO}}=L_{\mathrm{BC}}+L_{\mathrm{CO}}$ and $L_{\mathrm{OE}}=L_{\mathrm{OD}}+L_{\mathrm{DE}}$ are used to calculate the starting time $t_{\mathrm{B}}$ of the deceleration phase along $\overrightarrow{S_{1}}$, and the ending time $t_{\mathrm{E}}$ of the acceleration phase along $\overrightarrow{S_{2}}$.

Note that the curve $\mathrm{CD}$ is not a circular arc but a parabolic curve. Thus, the smoothed toolpath trajectory is not characterized by a radius of curvature. We rather quantify the smoothing of the toolpath by the maximal toolpath deviation $\varsigma$, which corresponds to the distance between the corner point $\mathrm{O}$ and the middle point $\mathrm{I}$ of the curve $\mathrm{CD}$ (see Fig. 2). The maximum deviation between the smoothed toolpath and the prescribed sharp trajectory is: (see derivation in Appendix C):

$$
\varsigma=\frac{\left[\kappa \min \left(V_{1}, V_{2}\right) \cos \left(\frac{\theta}{2}\right)\right]^{2}}{2 A_{\max } f\left(\frac{\alpha_{2}+\alpha_{1}}{2}\right)} .
$$

Hence, the maximum toolpath deviation is proportional to the square of the cruising printing speed and the square of the blending acceleration factor, and inversely proportional to the maximum acceleration. Moreover, the toolpath deviation is larger for acute angles and segments oriented in the diagonal directions than for obtuse angles and segments parallel to the $X$ or $Y$ axes.

The additional corner travelling time $\Delta t_{\mathrm{corner}, \kappa}$ due to the acceleration and deceleration phases during the turn is defined as the difference of the actual acceleration and deceleration time $\Delta t_{\kappa, \mathrm{BE}}$, and the hypothetical travelling time $\Delta t_{\text {ideal,BE }}$ along the exact toolpath at the full cruising speed (i.e. for the ideal turn with an instantaneous change of velocity). In the case where the two segments $\overrightarrow{S_{1}}$ and $\overrightarrow{S_{2}}$ forming the corner have the same cruising speed, $V_{1}=$ $V_{2}=V$, the additional corner traveling time is:

$$
\Delta t_{\text {corner }, \kappa} \equiv \Delta t_{\kappa, \mathrm{BE}}-\Delta t_{\text {ideal, BE }}=\frac{V}{A_{\max }}\left[\frac{(1-\kappa)^{2}}{2}\left(\frac{1}{f\left(\alpha_{1}\right)}+\frac{1}{f\left(\alpha_{2}\right)}\right)+\frac{2 \kappa(1-\kappa)}{f\left(\frac{\alpha_{2}+\alpha_{1}}{2}\right)} \cos \left(\frac{\theta}{2}\right)\right] .
$$

The general expression of $\Delta t_{\text {corner }, \kappa}$ where the two segments have different cruising speeds $V_{1} \neq V_{2}$ is derived in Appendix D. Successive segments with different cruising speeds may occur when the toolpath is made of short segments that do not have enough lengths for the printing head to accelerate to the cruising speed.

The maximal toolpath deviation and the additional corner travelling time does not only depend on the blending acceleration factor $\kappa$ and the angle $\theta$ of the corner, but also on the orientation of the corner with respect to the $X$ and $Y$ axes, which is reflected by the fact that the polar angles $\alpha_{1}$ and $\alpha_{2}$ of the two segments also appear in Eq. (15) and (16). Note that $\alpha_{1}, \alpha_{2}$, and $\theta$ are not independent variables, as they are linked by Eq. (11). The variations of the normalized toolpath deviation $\varsigma /\left(V^{2} / A_{\max }\right)$ and the normalized additional corner travelling time $\Delta t_{\text {corner }, \kappa} /\left(V / A_{\max }\right)$ with the blending acceleration factor $\kappa$ are represented in Fig. 4, for $90^{\circ}$ and $30^{\circ}$ turns with different orientations, where $V_{1}=V_{2}=V$. It can be seen in Fig. 4 that the additional corner travelling time decreases monotonously with the blending acceleration factor, while the maximal toolpath deviation monotonously increases. 
For turns with fully blended accelerations $(\kappa=1)$, the printing head travels as quickly as for the ideal turn; $\Delta t_{\text {corner }, k=1}=0$. Eq. (15) and (16), as well as the graphs in Fig. 4, are useful to identify the appropriate value of the blended acceleration factor, according to the desired compromise between the printing time and the accuracy of the toolpath.
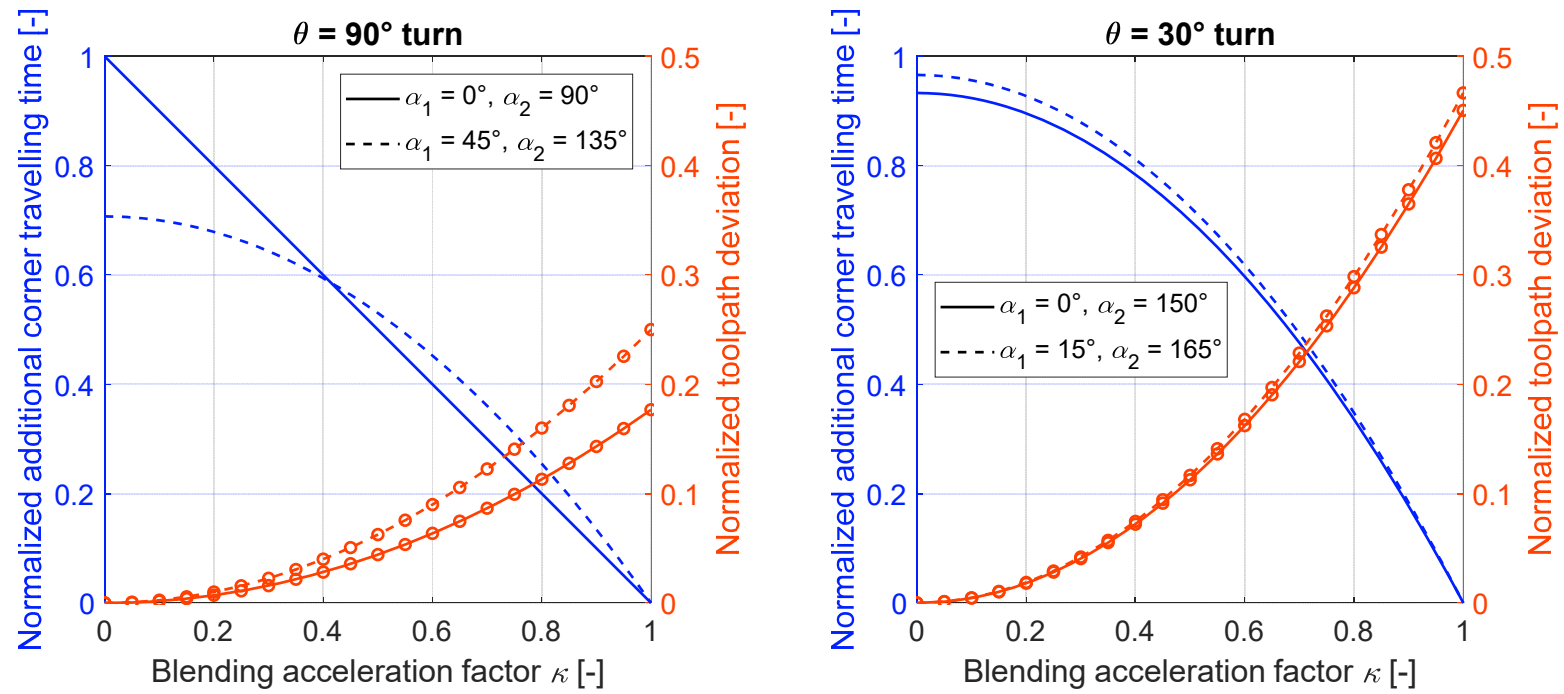

Fig. 4. Normalized additional corner travelling time $\Delta t_{\text {corner, } \kappa} /\left(V / A_{\max }\right)$ and normalized maximal toolpath deviation $\varsigma /\left(V^{2} / A_{\max }\right)$ versus the blending acceleration factor $\kappa$, where $V_{1}=V_{2}=V$, for $90^{\circ}$ and $30^{\circ}$ turns with different orientations. The solid lines represent the cases where the first segment is aligned with the $X$ axis $\left(\alpha_{1}=\right.$ $0^{\circ}, \alpha_{2}=\pi-\theta$ ), while the dashed lines represent the cases where the bisector angle of the corner is aligned with the $X$ axis $\left(\alpha_{1}=\theta / 2, \alpha_{2}=\pi-\theta / 2\right)$.

\subsection{Extrusion rate}

For each linear segment $\overrightarrow{S_{l}}$, the G-code also specifies the length $\delta_{i}$ of feeding filament to push inside the liquefier. Assuming that the printing head travels along the segment $\overrightarrow{S_{l}}$ with the average speed $V_{i}$ specified in the G-code, the travelling time along the segment is

$$
\Delta t_{\overrightarrow{S_{l}}}=L_{i} / V_{i}
$$

Then, the feeding speed $V_{f}$ of the filament pushed into the liquefier is

$$
V_{f}=\delta_{i} / \Delta t_{\vec{S}_{i}} \text {. }
$$

By virtue of mass conservation, the mass flow rate of extrusion (i.e. the quantity of mass extruded per unit of time) equals the mass flow rate of the feeding filament entering the liquefier. Thus, the extrusion volumetric flux $U$ (i.e. the average velocity of the molten plastic extruded from the nozzle orifice) relates to $V_{f}$ as:

$$
U=\phi V_{f}\left(\frac{D_{f}}{D}\right)^{2}(1+\beta \Delta T),
$$

where $D_{f}$ is the average diameter of the feeding filament, $D$ is the diameter of the extrusion nozzle, $\phi$ is the extrusion multiplier, $\beta$ is the volumetric thermal expansion coefficient of the polymer, and $\Delta T=T_{\text {ext }}-T_{0}$ is the difference between the extrusion temperature $T_{\text {ext }}$ and the room temperature $T_{0}$. Here, the extrusion multiplier is seen as an empirical calibration factor that corrects the theoretical value of $V_{f}$ for the variability of the filament cross-section, the potential slippage of the feeding rollers onto the filament, or any other source of errors [26].

As shown in [25], the width of the deposited strand depends on the ratio $V / U$ of the printing speed to the extrusion volumetric flux. Therefore, the strand's width is affected by the deceleration and acceleration of the printing head during the turn, unless the extrusion volumetric flux is maintained proportional to the printing speed. However, in practice the extrusion rate is normally kept constant through the entire segments, in spite of the fact that it leads to material over-deposition at the corners.

The feasibility of synchronizing the filament feeding rate with the printing speed in material extrusion additive manufacturing was investigated in $[9,10]$. The synchronization of the extrusion rate poses certain difficulties because of the lack of an accurate dynamical model that can predict the response time of the extruder, and the absence of active feedback control of the filament feeding mechanism [13]. The variations in the filament feeding rate affect the residence time of the plastic and the position of the melting line inside the liquefier. Moreover, the required force to extrude the material varies non-linearly with the extrusion rate, because of the non-Newtonian (temperature-dependent and shear-thinning) rheological behavior of the molten polymer [20,21,32,33]. Furthermore, we expect that quick variations in the filament feeding rate (and the liquefier's pressure) increase the risk of filament slippage and nozzle clogging. However, addressing the difficulty of implementing a synchronizing 
extrusion rate is outside the scope of our current investigations. Therefore, we have assumed in the numerical simulations that the extrusion rate can be successfully synchronized with the printing speed. For that reason, the numerical model only includes the exit section of the extrusion nozzle, and directly takes the extrusion volumetric flux $U$ as a model input. The corresponding values of $\delta_{i}$ that would be specified in a G-code to reproduce the extrusion simulation in an experiment can be recover from Eq. (17) to (19).

\section{Numerical simulations}

The numerical model is used to simulate the deposition flow and the strand forming at corners with $90^{\circ}$ and $30^{\circ}$ angles, with constant extrusion rates and proportional extrusion rates. In each case, we first simulated the ideal turn (with instantaneous change of speed direction), and the partially blended acceleration turns, for $\kappa=0,0.1, \ldots$ 1 , for two maximum accelerations $A_{\max }=1000 \mathrm{~mm} / \mathrm{s}^{2}$ and $A_{\max }=2000 \mathrm{~mm} / \mathrm{s}^{2}$. The cruising speed was $V=60 \mathrm{~mm} / \mathrm{s}$ and the velocity ratio was $V / U=1$. The results of the numerical simulations were post-processed in order to quantify the amount of underfill and overfill of deposited material for the different cases, as compared to the ideal turns.

Material deposition along a straight line was also simulated, for different velocity ratios $V / U$, in order to validate the predictions of the CFD model with experimental data previously published [26]. Moreover, the accuracy of the numerical results were assessed via a mesh refinement analysis with two mesh sizes. A summary of all the numerical parameters used in the simulations is provided in Table 1.

\subsection{Computational model}

The geometry of the computational model consists of the tip of a cylindrical extrusion nozzle and the build volume between the printing head and the build plate. The extrusion nozzle has a diameter $D=0.4 \mathrm{~mm}$, and is position at the distance $h=0.32 \mathrm{~mm}$ above the build plate, corresponding to the layer height; see Fig. 5 . For the $90^{\circ}$ turn, the build volume is a rectangular cuboid of $3.8 \mathrm{~mm} \times 5.8 \mathrm{~mm} \times 0.48 \mathrm{~mm}$. For the $30^{\circ}$ turn, the build volume is a truncated rectangular cuboid of $7.2 \mathrm{~mm} \times 4.1 \mathrm{~mm} \times 0.48 \mathrm{~mm}$ with a chamfer at $30^{\circ}$ angle; see Fig. 5 . The simulations of material extrusion along a straight line use a smaller geometry with a build volume of $4.5 \mathrm{~mm} \times 1.6$ $\mathrm{mm} \times 0.48 \mathrm{~mm}$.

Table 1. Summary of the numerical parameters used in the simulations.

\begin{tabular}{|c|c|c|c|}
\hline Parameter & Symbol & Unit & Values \\
\hline \multicolumn{4}{|c|}{ Motion planning controls } \\
\hline Angle of the turn ${ }^{\dagger}$ & $\theta$ & $\circ$ & 30,90 \\
\hline Cruising printing speed & V & $\mathrm{mm} / \mathrm{s}$ & $60,45^{\ddagger}, 30^{\ddagger}$ \\
\hline Steady extrusion volumetric flux & $U$ & $\mathrm{~mm} / \mathrm{s}$ & 60 \\
\hline Maximum acceleration ${ }^{\dagger}$ & $A_{\max }$ & $\mathrm{mm} / \mathrm{s}^{2}$ & 1000,2000 \\
\hline Blending acceleration factor ${ }^{\dagger}$ & $\kappa$ & - & $0,0.1, \ldots 1$ \\
\hline \multicolumn{4}{|c|}{ Geometrical parameters } \\
\hline Nozzle diameter & $D$ & $\mathrm{~mm}$ & 0.4 \\
\hline Layer height & $h$ & $\mathrm{~mm}$ & 0.32 \\
\hline \multicolumn{4}{|c|}{ Material properties } \\
\hline Polymer density & $\rho$ & $\mathrm{kg} / \mathrm{m}^{3}$ & 1250 \\
\hline Polymer viscosity & $\eta$ & Pa.s & 1000 \\
\hline Density of air & $\rho_{\text {air }}$ & $\mathrm{kg} / \mathrm{m}^{3}$ & 0.01 \\
\hline Viscosity of air & $\eta_{\text {air }}$ & $\mathrm{Pa} \cdot \mathrm{S}$ & 0.01 \\
\hline Gravitational acceleration & $g_{0}$ & $\mathrm{~m} / \mathrm{s}^{2}$ & 9.81 \\
\hline \multicolumn{4}{|c|}{ Simulation parameters } \\
\hline Cartesian grid spacing & $\delta l$ & $\mu \mathrm{m}$ & 20 (mesh M1), 10‡ (mesh M2) \\
\hline Time-step increment & $\delta t$ & $\mathrm{~ms}$ & 0.1 (mesh M1), $0.05^{\ddagger}($ mesh M2) \\
\hline Maximal Courant number & $C$ & - & 0.3 \\
\hline
\end{tabular}

In the simulations, the nozzle is static, while the build plate moves with the prescribed velocity components $-V_{x}(t)$ and $-V_{y}(t)$ along the $X$ and $Y$ axes. The tangential velocity of the build plate simulates the motion of the printing head, according to the desired toolpath planning. The top surface of the extrusion nozzle (represented in yellow in Fig. 5) is an inlet boundary with a fully developed velocity profile with the average extrusion volumetric flux $U=60 \mathrm{~mm} / \mathrm{s}$. Away from the corner, the printing head moves along the linear segments with the cruising speed $V=60 \mathrm{~mm} / \mathrm{s}$ (printing speeds of $45 \mathrm{~mm} / \mathrm{s}$ and $30 \mathrm{~mm} / \mathrm{s}$ are also used for the simulations of material deposition 
along a straight line). No-slip boundary conditions are applied on the solid walls, so that the material sticks to the nozzle wall and the build plate. Other boundaries are outlets, where the material is free to exit the computational domain.

The deposition flow of the extruded material is modeled as an isothermal incompressible Newtonian fluid flow, similarly to [25,26]. Serdeczny et al. [26] have shown that those assumptions are sufficient to predict the shape of the deposited strands observed in experiments. Moreover, numerical simulations with power-law constitutive models [34] showed that shear-thinning has virtually no effect on the strand forming, because the free-surface deposition flow is not subjected to shear deformation (in contrast with the flow inside the liquefier-extruder). In the current work, the flow is governed by the Navier-Stokes equations, which account for the mass and momentum conservation.

The typical deposition flows of plastic materials have very low Reynolds numbers, $\operatorname{Re}=\rho U D / \eta \sim 10^{-5}$ (which compares the magnitude of inertial effects to viscous stresses), where $\rho$ and $\mu$ are the density and viscosity of the molten polymer, respectively. In addition, the deposition flows typically have Froude numbers around the unity, $\mathrm{Fr}=U / \sqrt{g_{0} h} \sim 1$ (which compares the magnitude of inertial effects to gravity), where $g_{0}$ is the gravitational acceleration on Earth. Hence, both gravity and inertial effects are negligible compared to the internal viscous forces of the flow. Consequently, the material deposition is a creeping flow that does not depend on the actual values of viscosity and density, as long as $R e \ll 1$. However, for the sake of completeness, the material properties used in the simulations are included in Table 1 . The molten polymer has a density $\rho=1250 \mathrm{~kg} / \mathrm{m}^{3}$ and a viscosity $\eta=1000 \mathrm{~Pa} \cdot \mathrm{s}$. The density and viscosity of the surrounding air (which is also included in the simulations) are set to the values $\rho_{\text {air }}$ $=0.01 \mathrm{~kg} / \mathrm{m}^{3}$ and $\eta_{\text {air }}=0.01 \mathrm{~Pa} \cdot \mathrm{s}$, respectively. As the actual flow of air is not the interest of this study, the air is assigned artificial material properties, such that it facilitates numerical convergence, without affecting the flow of the molten polymer.

(a)

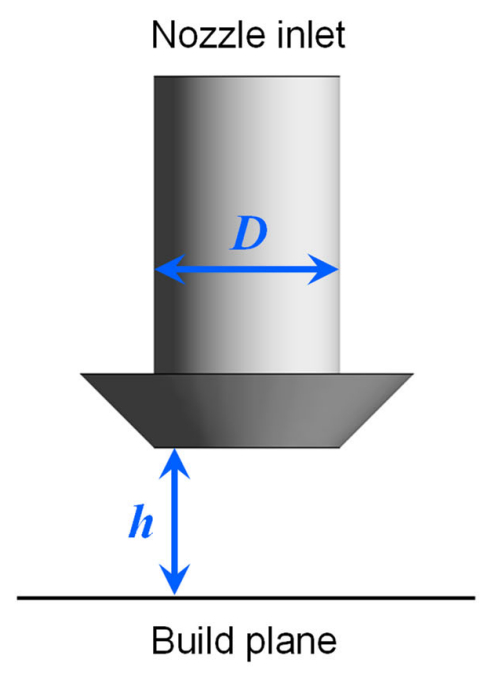

(b)

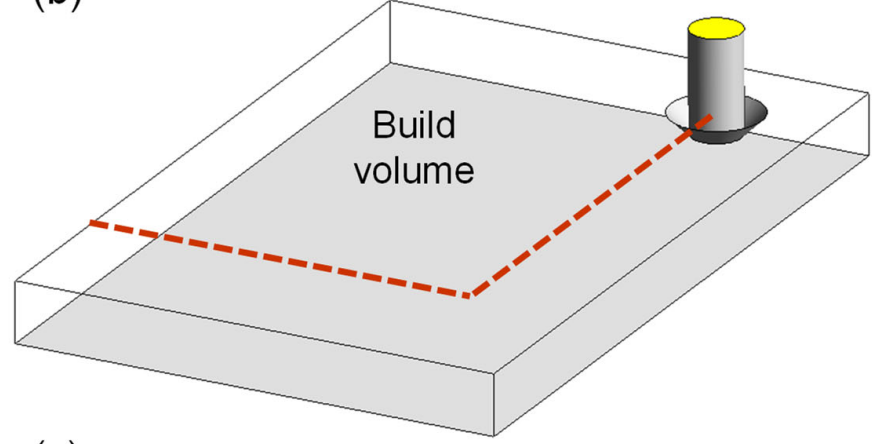

(c)

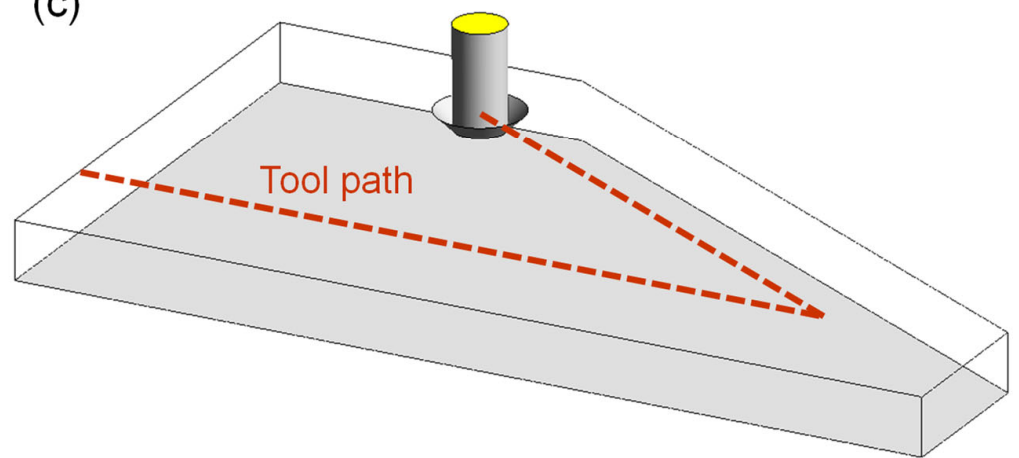

Fig. 5. (a) Geometry of the extrusion nozzle; (b) computational domain for the $90^{\circ}$ turn; (c) computational domain for the $30^{\circ}$ turn.

\subsection{Numerical methods}

The deposition flow was solved with the general-purpose CFD software ANSYS ${ }^{\circledR}$ Fluent R18.2. The free surface flow was simulated as a multi-phase flow of two immiscible fluids (molten plastic and air), using the volume-of-fluid interface capturing method. Reviews of geometric and algebraic volume-of-fluid schemes can be found in [35-37]. The computational domain was meshed using a Cartesian cut-cell mesh with a grid spacing $\delta l=20 \mu \mathrm{m}$ (mesh M1), resulting in about 1.3 million grid cells, in both geometries for the $30^{\circ}$ and $90^{\circ}$ turns. A twice refined mesh, with grid spacing $\delta l=10 \mu \mathrm{m}$ (mesh M2), was also used for the simulations of material extrusion along a straight line. The computational domain of the straight line deposition had approximately 440,000 grid cells with mesh M1, and 3.5 million grid cells with mesh M2. 
The momentum equations are discretized with collocated control volumes, using a coupled pressure-velocity scheme and the PRESTO scheme for the pressure discretization. Gradients were evaluated with the Green-Gauss node-based scheme. The convective terms were discretized with the QUICK scheme. The volume fraction of the molten plastic inside the control volumes was solved with the implicit modified HRIC scheme [38]. The apparent material properties of the control volumes were averaged according to the volume fractions of molten plastic and air. The transient governing equations of the flow were discretized with the first order implicit scheme. The timestep increment was set to $\delta t=0.1 \mathrm{~ms}$ for the mesh M1, and $\delta t=0.05 \mathrm{~ms}$ for the mesh $\mathrm{M} 2$, such that the corresponding maximal Courant number was $C=0.3$, in all the simulations.

\subsection{Post-processing of the results}

At the end of numerical simulations, the position of the free-surface was reconstructed via interpolation of the 0.5-isolevel of the plastic volume fraction, along the grid lines of the Cartesian mesh. The reconstructed free-surfaces were saved as triangulated surface in the STL file format. The surfaces were edited using the stlTools MATLAB toolbox [39], to trim and recenter the geometries around the corner. The open faces of the STL surfaces (after the trimming operation) were patched with additional triangles, calculated by polygon triangulation [40]. At that point, the STL surfaces possessed a very large number of vertices and triangles (due to the fine Cartesian mesh used in the numerical simulation), which makes it impractical to perform Boolean operations on the geometries. The surface meshes were simplified by quadratic edge collapse decimation using the open-source software MeshLab [41], in order to reduce the total number of triangles to about 10,000. The resulting surface meshes were further cleaned and repaired using several MeshLab filters, to remove non-manifold vertices, non-manifold edges, self-intersecting faces, and to close holes, until the geometries were completely manifold and watertight.

Afterwards, the repaired surface meshes were imported to the open-source software Gmsh [42], to perform geometrical Boolean operations. The 3D solids of the deposited material at the inside and the outside of the corner, $\mathbb{S}_{\text {inside }}$ and $\mathbb{S}_{\text {outside, }}$ were defined as follow:

$$
\begin{gathered}
\mathbb{S}_{\text {inside }}=\mathbb{S}_{\text {strand }} \cap \mathbb{S}_{\text {primitive }} \\
\mathbb{S}_{\text {outside }}=\mathbb{S}_{\text {strand }}-\mathbb{S}_{\text {primitive }}
\end{gathered}
$$

where $\mathbb{S}_{\text {strand }}$ is the 3D solid bounded by a STL surface mesh of the deposited material, and $\mathbb{S}_{\text {primitive }}$ is a solid primitive of the corner; see Fig. 6. Finally, the 3D solids $\mathbb{S}_{\text {inside }}$ and $\mathbb{S}_{\text {outside }}$ were meshed with 3D tetrahedral meshes, in order to calculate their volumes. The volumes of each solid $\mathbb{S}$ was calculated with the following formulas:

$$
V(\mathbb{S})=\frac{1}{6} \sum_{i=1}^{N}\left|\left(\boldsymbol{x}_{k, 2}-\boldsymbol{x}_{k, 1}\right) \cdot\left[\left(\boldsymbol{x}_{k, 3}-\boldsymbol{x}_{k, 1}\right) \times\left(\boldsymbol{x}_{k, 4}-\boldsymbol{x}_{k, 1}\right)\right]\right|
$$

where $\boldsymbol{x}_{k, 1}, \boldsymbol{x}_{k, 2}, \boldsymbol{x}_{k, 3}$ and $\boldsymbol{x}_{k, 4}$ are the coordinate vectors of the 4 vertices defining the $k^{\text {th }}$ tetrahedron (the ordering of the vertices has no importance), and $N$ is the total number of tetrahedra of the 3D mesh.

The amount of overfill and underfill material deposited at the corner was evaluated by comparison with the simulated strands deposited with the ideal turns. The overfill/underfill volumes at the inside and outside of the turn, $V_{\text {inside }}^{\kappa, A_{\max }}$ and $V_{\text {outside }}^{\kappa, A_{\max }}$, respectively, for the blending acceleration factor $\kappa$ and the maximum acceleration $A_{\text {max }}$ are calculated as:

$$
\begin{gathered}
V_{\text {inside }}^{\kappa, A_{\max }}=V\left(\mathbb{S}_{\text {inside }}^{\kappa, A_{\max }}\right)-V\left(\mathbb{S}_{\text {inside }}^{\text {ideal }}\right), \\
V_{\text {outside }}^{\kappa, A_{\max }}=V\left(\mathbb{S}_{\text {outside }}^{\kappa, A_{\max }}\right)-V\left(\mathbb{S}_{\text {outside }}^{\text {ideal }}\right)
\end{gathered}
$$

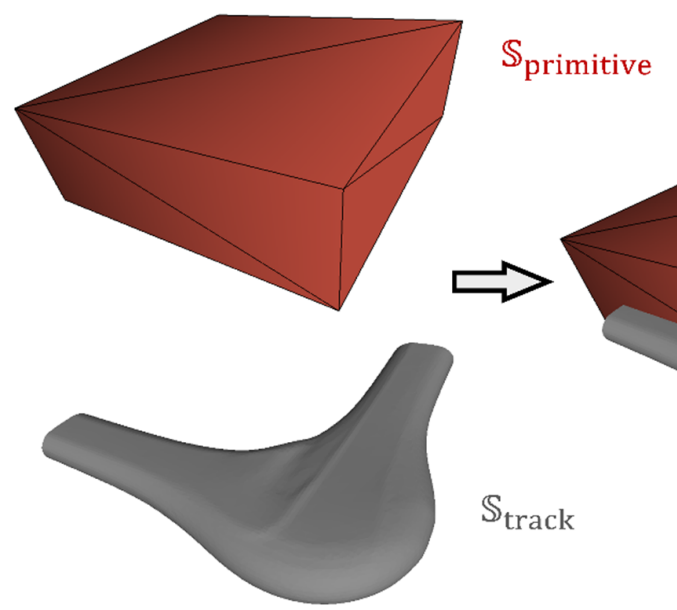

Fig. 6. 3D solids of the material at the inside and outside of the corner, obtained by geometrical Boolean operations between the 3D solids of the deposited strand and the corner primitive. 


\section{Results and discussions}

\subsection{Model validation}

As mentioned in Section 2, the shape of printed corners does not only depend on the toolpath trajectory and the nominal extrusion rate, but also on the vibrations of the positioning system and possible over- and under-extrusion due to the transient responses of the extruder. Those two phenomena are generally uncontrolled during experiments, and have been neglected in the present model. Thus, the CFD model of material deposition was validated with the extrusion simulations along a straight line, with a steady printing head motion. The numerical results are compared with experimental data previously published in [26], where straight strands were printed with different printing speeds and layer heights. Before the experiments, the extrusion rate of the 3D printer was carefully calibrated, and the layer height was corrected to compensate for planarity errors of the build plane. The height and width of the printed strands were measured from optical micrographs of polished cross-sections [26].

Fig. 7 provides a comparison of the numerical and experimental results, for three different values of the velocity ratio, $V / U=0.5,0.75$ and 1 . The graphs of the normalized strand height $t / D$ and the normalized strand width $W / D$ in Fig. 7 (a) show that the numerical results agree quantitatively well with the experimental data. Moreover, the two mesh resolutions produce sensibly the same results. The numerical results are also compared with two analytical solutions of the strand profile based on the volume conservation [25], assuming elliptic and oblong cross-sections. Those analytical solutions deviate from both the experiments data and the results of the CFD simulations. Snapshots of the simulated material deposition in Fig. 7 (b) confirm that the cross-section area and the strand width increase when the velocity ratio $V / U$ is reduced. Fig. 7 (c) compares the shape of measured cross-sections with the simulation results, for the meshes M1 and M2. The predicted strand profiles with the two mesh resolutions are almost identical. Moreover, the computational time with the mesh M1 is reduced by a factor of 10 compared to the computational time with the mesh M2. Thus, we concluded that the mesh M1 represents a good compromise between numerical precision and computational cost. Consequently, all the following simulations of the material deposition along corners were simulated with the mesh M1. 
(a)

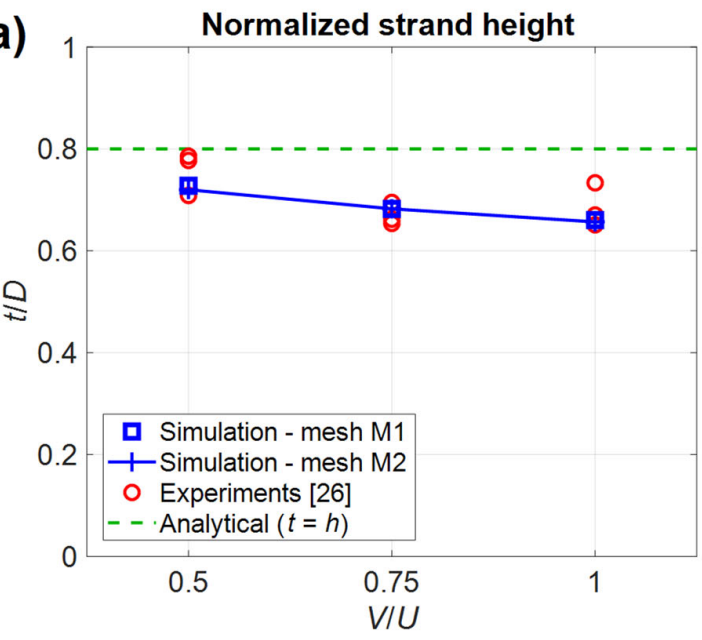

(b)
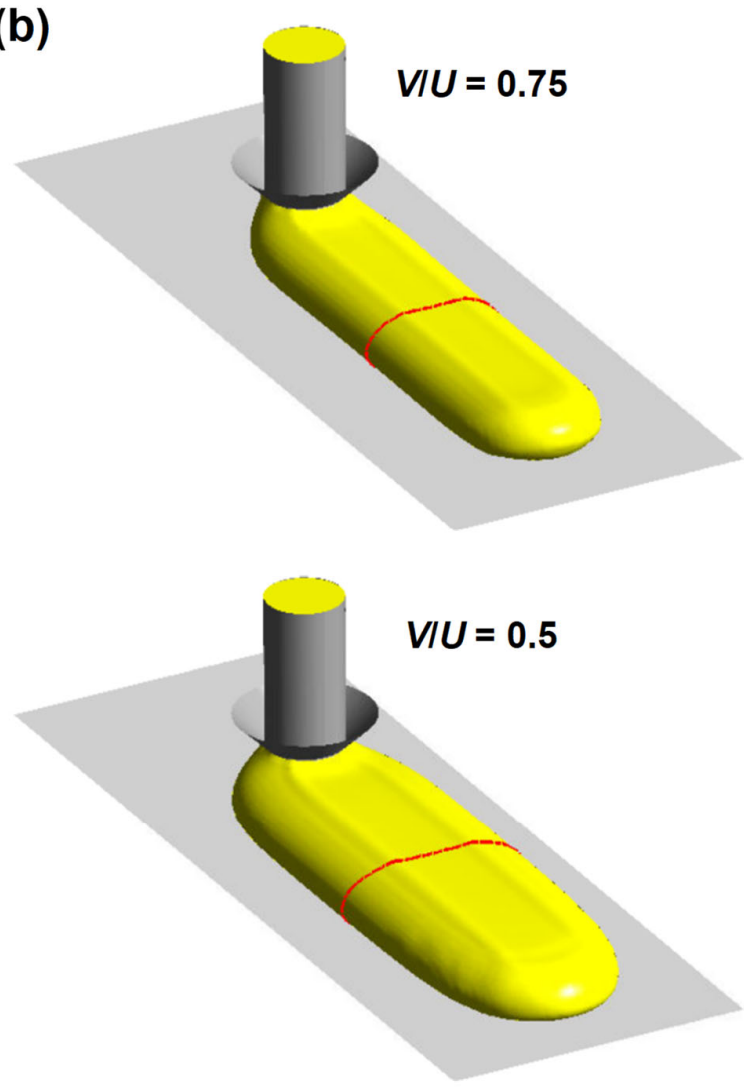

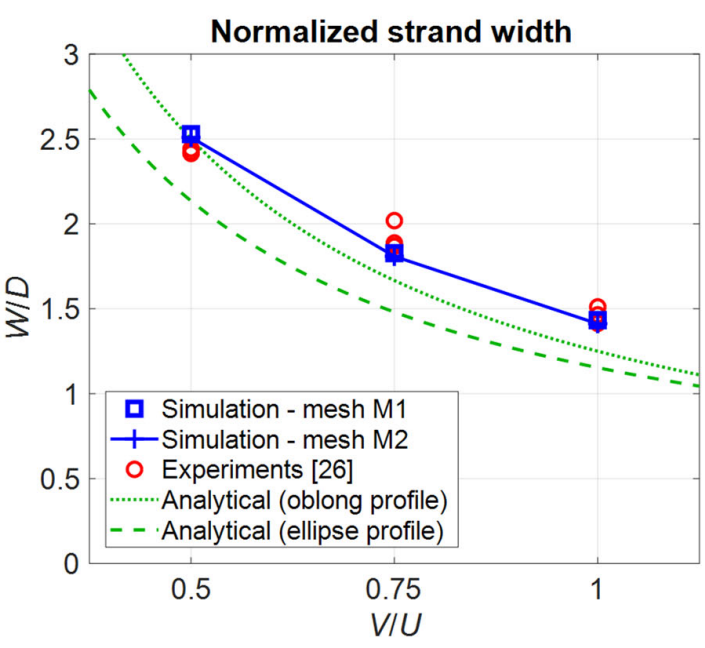

(c)

$$
h / D=0.8, V / U=1
$$

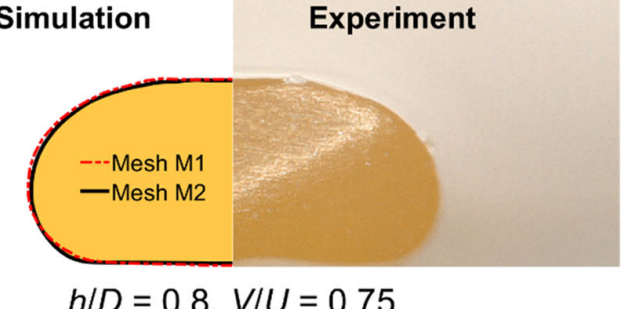

$h / D=0.8, V / U=0.75$

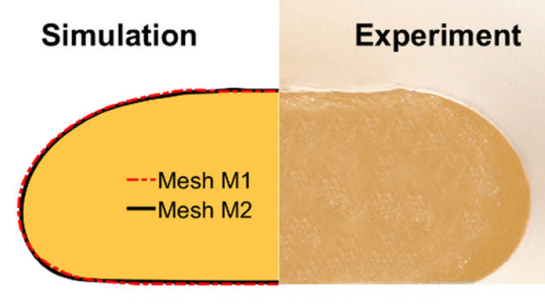

$h / D=0.8, V / U=0.5$

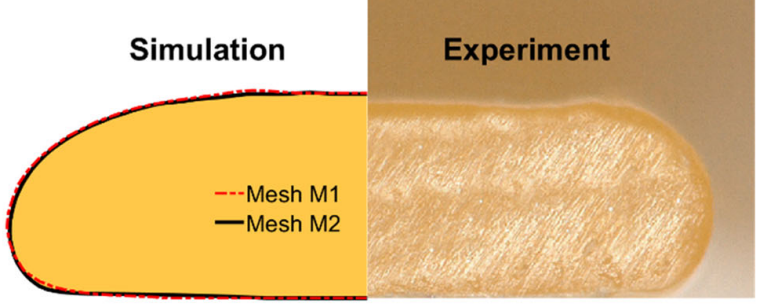

Fig. 7. Comparison of the simulated strand profiles deposited along a straight line with the experimental data in [26], for different values of the velocity ratio $V / U$.

\subsection{Ideal turn}

The shapes of the strands deposited with ideal turns (instantaneous change of speed direction) for the $90^{\circ}$ and $30^{\circ}$ corners are displayed in Fig. 8. The printing head motion with ideal turns follows exactly the prescribed toolpaths of the sharp corners. However, we observe that the resulting corners have a small amount of corner rounding and corner swelling, which is due to the facts that the deposited strand has an elliptical cross-section, and the strand gets formed slightly behind the moving printing head (due to the travel time for the extruded material to reach the substrate, as shown in Fig. 9). In our simulations, the distance between the position of the nozzle and the 
strand formation is about $\varepsilon \approx 0.28 D$. This distance is expected to depend on the velocity ratio $V / U$ and the normalized layer height $h / D$.

Fig. 10 shows horizontal cuts of the deposited strands, though their mid-planes, and a cross-sectional cut far away from the corner. The simulated strands of the ideal turns are compared with the oblong analytical shape which is commonly used in slicer software. The oblong analytical shape assumes that the strand height $t$ equals the layer height $h$. Thus, the analytical strand width $W$ is given by [25]:

$$
W=\frac{\pi}{4} \frac{U}{V} \frac{D^{2}}{h}+\left(1-\frac{\pi}{4}\right) h
$$

As observed in the sub-section 5.1, the simulated strand profile has a lower strand height $t$ and a larger strand width $W$ than the analytical shape. On the horizontal cuts, the analytical shape presents an area of underfill $A_{\text {underfill }}$ at the outside of the turn, and an area of double deposition $A_{\text {double_deposition }}$ at the inside of the turn; see Fig. 10 . The analytical area of underfill and double deposition are identical $[2,8]$ :

$$
A_{\text {underfill }}=A_{\text {double_deposition }}=\frac{W^{2}}{4}\left(\cot \left(\frac{\theta}{2}\right)+\frac{\theta}{2}-\frac{\pi}{2}\right) \text {. }
$$

The volume corresponding to the area of double deposition must be redistributed at the outskirt of the strand (not accounted to in the analytical shape); which explains that the corners have a larger radius in the simulations. Moreover, the distance $\varepsilon$ between the position of material extrusion and the position of strand forming (see Fig. 9) also contributes to produce corner rounding, in a similar way as show in [43], despite the sharp toolpath of the ideal turn.
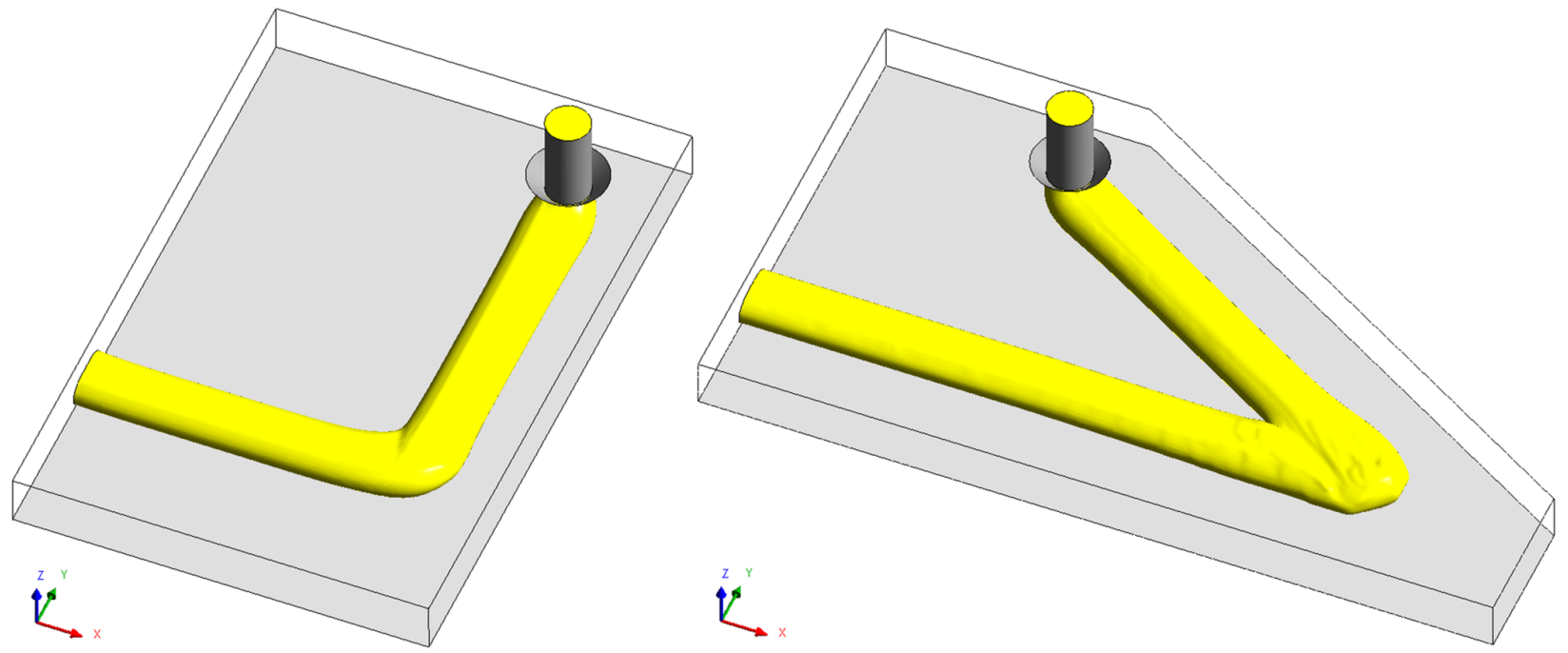

Fig. 8. Ideal material deposition for the $90^{\circ}$ and $30^{\circ}$ turns with instantaneous changes of speed direction.

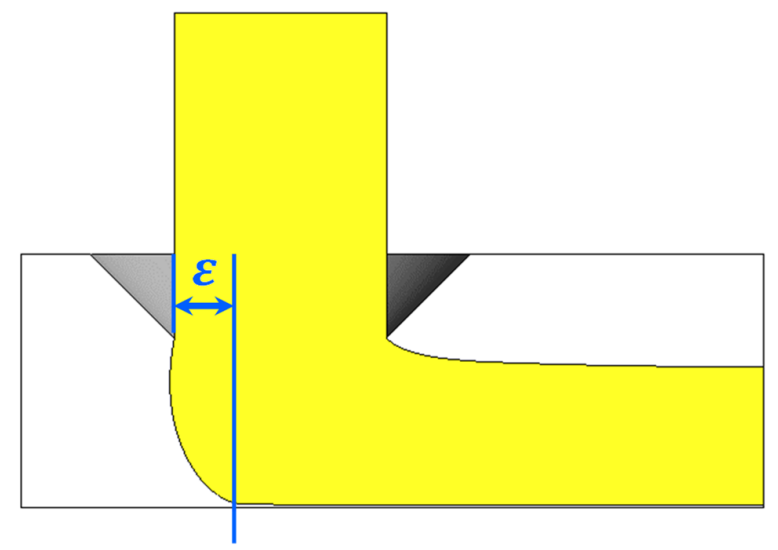

Fig. 9. Transversal cut of the extruded material. The strand gets formed at a distance $\varepsilon$ behind the extrusion nozzle. 

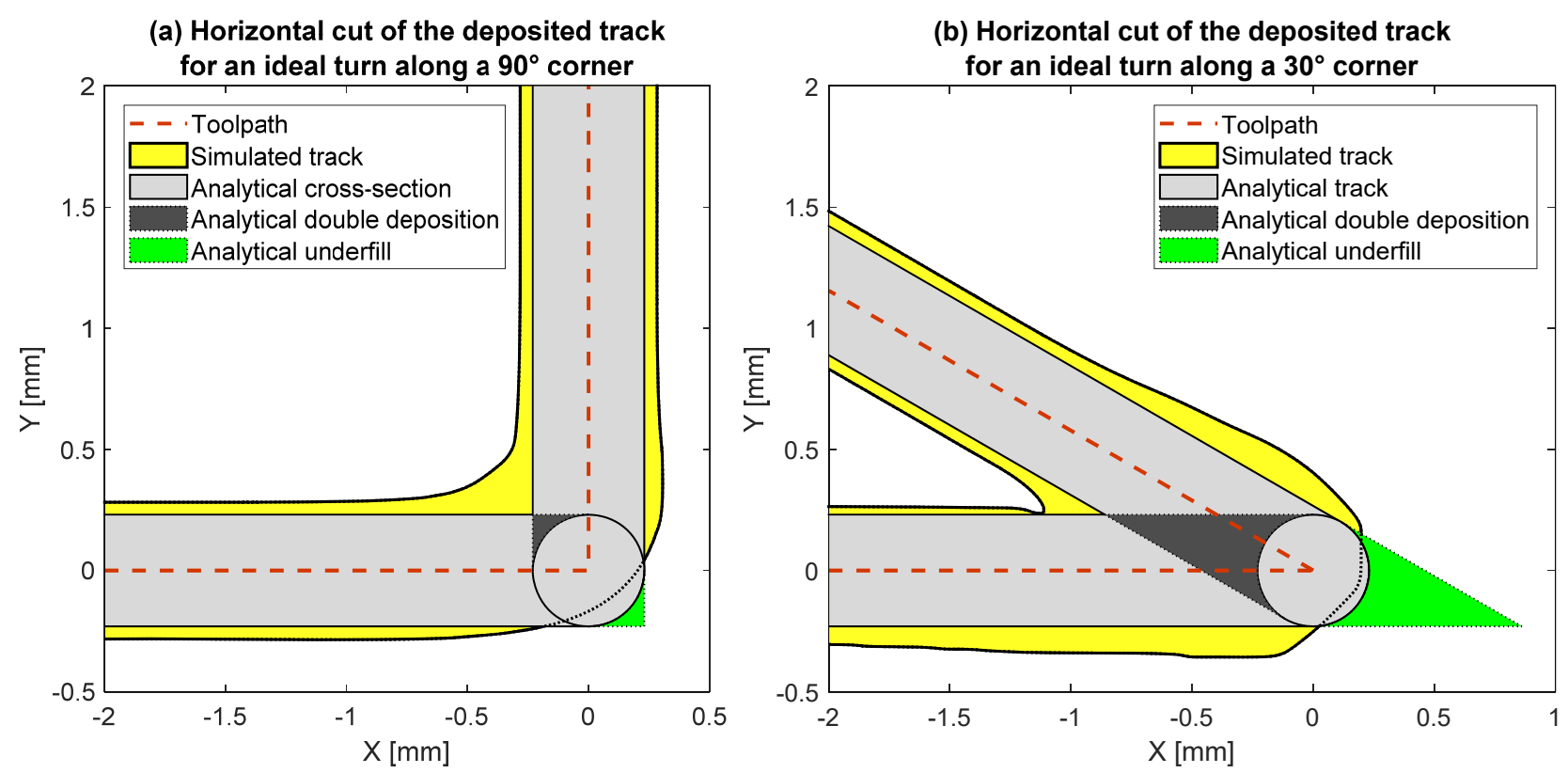

(c) Cross-sectional cut of the deposited track

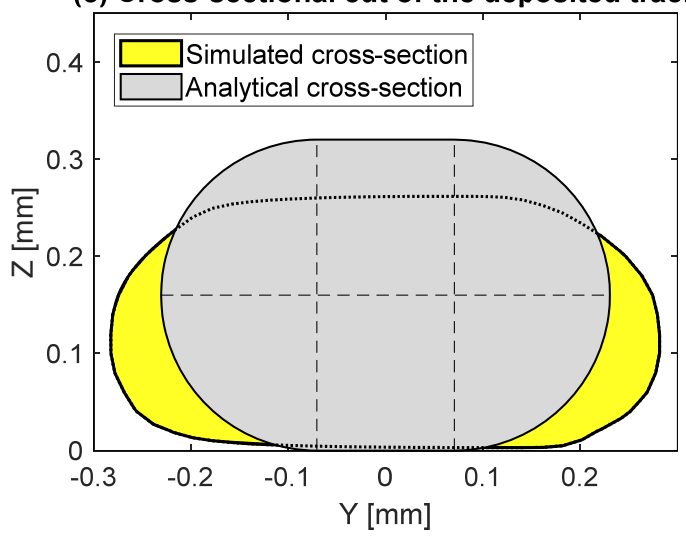

Fig. 10. Comparison of analytical and simulated strands deposited with the ideal turns; (a) horizontal cut of the $90^{\circ}$ corner; (b) horizontal cut of the $30^{\circ}$ corner; (c) cross-sectional cut far away from the corner.

\subsection{Constant extrusion rate}

Top views of the simulated corner prints are represented in Fig. 11 for the $90^{\circ}$ corners and in Fig. 12 for the $30^{\circ}$ corners, for $A_{\max }=1000 \mathrm{~mm} / \mathrm{s}^{2}$ and different values of the blending acceleration factor. The printed corners present significant swelling due to over-extrusion, when $\kappa=0$ (stop-at-turn toolpath). On the other hand, the corners exhibit a smooth rounding, when $\kappa=1$ (fully blended accelerations toolpath). The scenario where $\kappa=0.5$ and $\kappa=0.8$ (partially blended accelerations toolpaths) correspond to intermediate cases, where some of the over-extrusion compensates for the rounding of the toolpath.

Fig. 13 and Fig. 14 represent the evolution of the amount of overfill and underfill at the inside and outside of the corner, as a function of the blending acceleration factor. Here, the amount of overfill and underfill are calculated as compared to the simulations with ideal turns (infinite acceleration). In both cases of the $90^{\circ}$ and $30^{\circ}$ turns, the amount of overfill at the outside of the corner decreases monotonically when the blending acceleration factor increases. The reduction of overfill eventually yields underfill at the outside of the corner, when $\kappa$ exceeds a value of around 0.8 or 0.9 , depending on the maximum acceleration and the angle of the turn. For the $90^{\circ}$ corner, the amount of overfill at the inside of the corner also decreases with the increase of the blending acceleration factor, when $0<\kappa<0.8$, due to the overall reduction of over-deposition. However, the amount of overfill at the inside of the corner increases with the blending acceleration factor, when $0.8<\kappa<1$, because the corner rounding deviates the material deposition from the ideal trajectory towards the inside of the turn. For the $30^{\circ}$ turn, the overfill at the inside of the corner increases monotonically with the blending acceleration factor, because the reduction of overextrusion with increasing $\kappa$ is mitigated by the deviation of the material deposition towards the inside of the corner (corner rounding). 

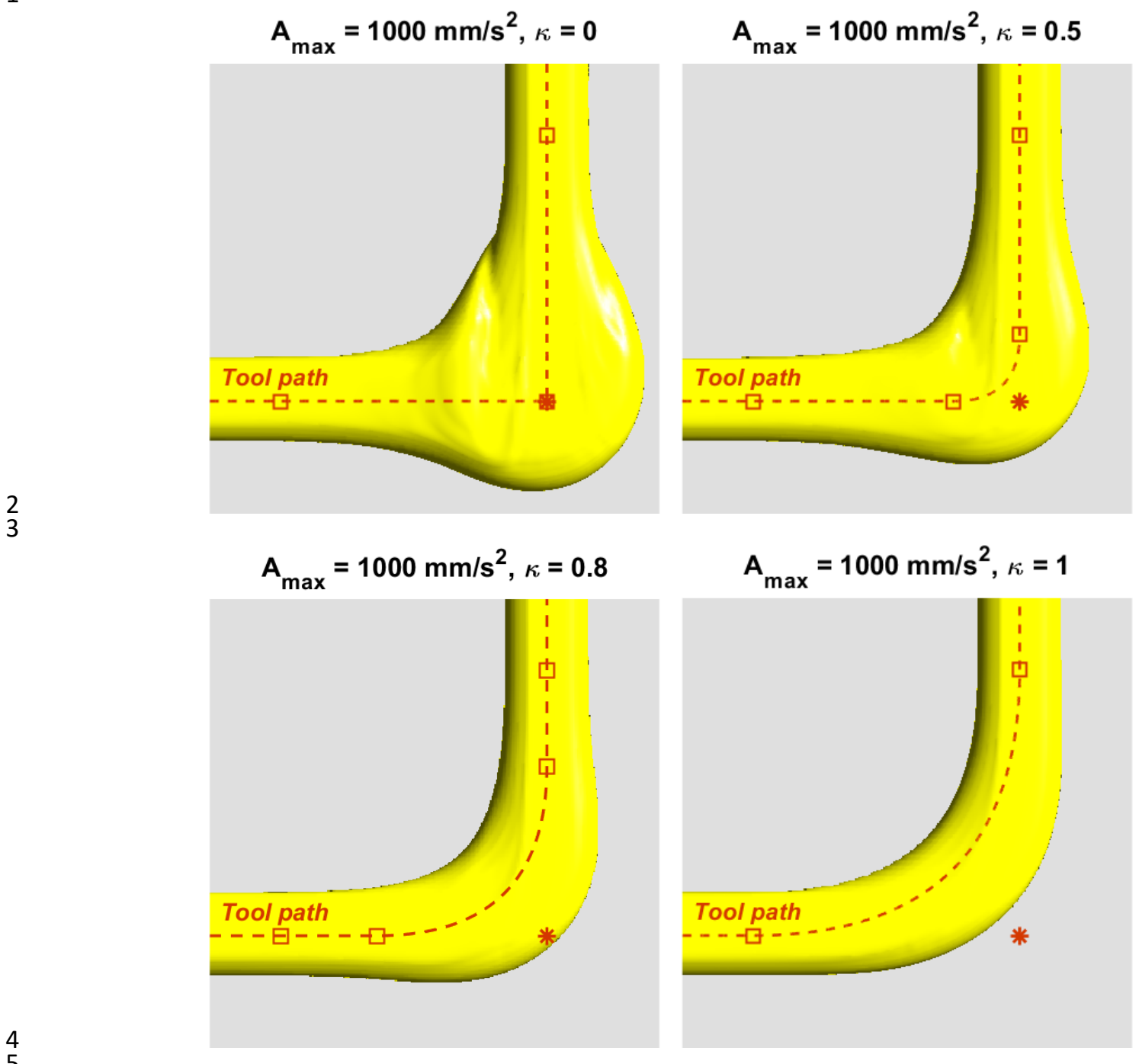

Fig. 11. Top views of $90^{\circ}$ corners, printed with different blending acceleration factors $\kappa$ and a constant extrusion rate.

$$
A_{\max }=1000 \mathrm{~mm} / \mathrm{s}^{2}, \kappa=0
$$

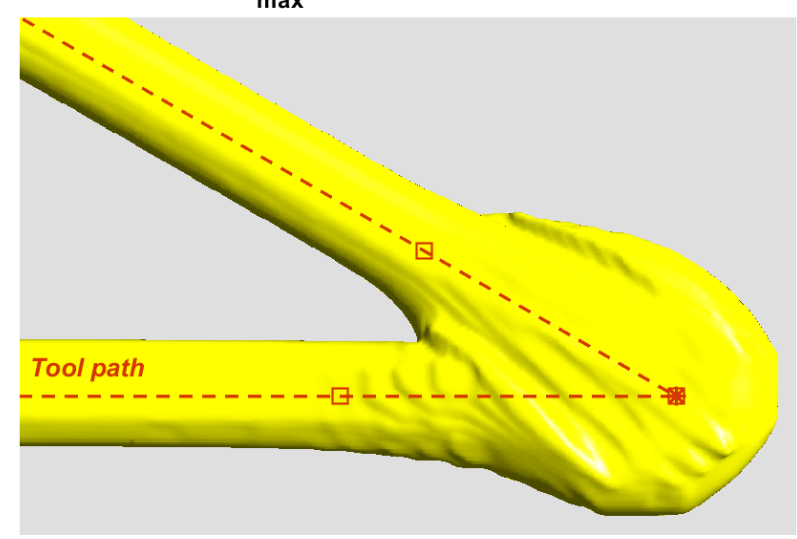

$A_{\max }=1000 \mathrm{~mm} / \mathrm{s}^{2}, \kappa=0.5$

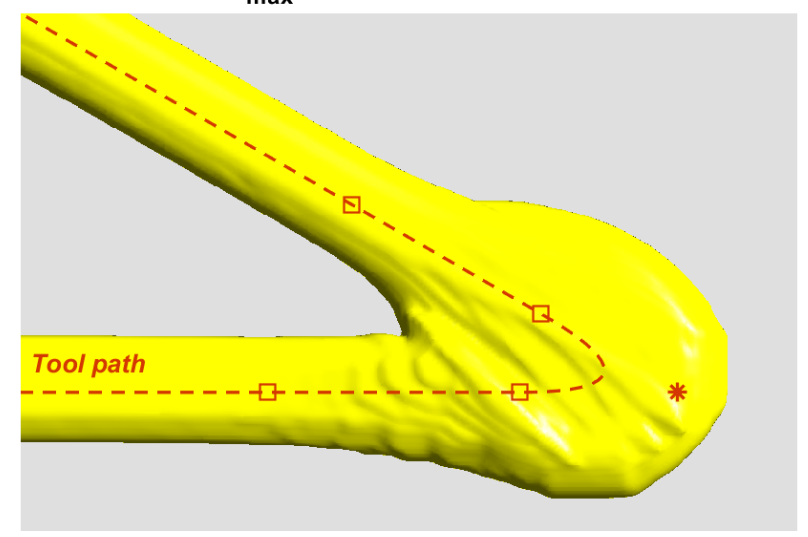


$A_{\max }=1000 \mathrm{~mm} / \mathrm{s}^{2}, \kappa=0.8$

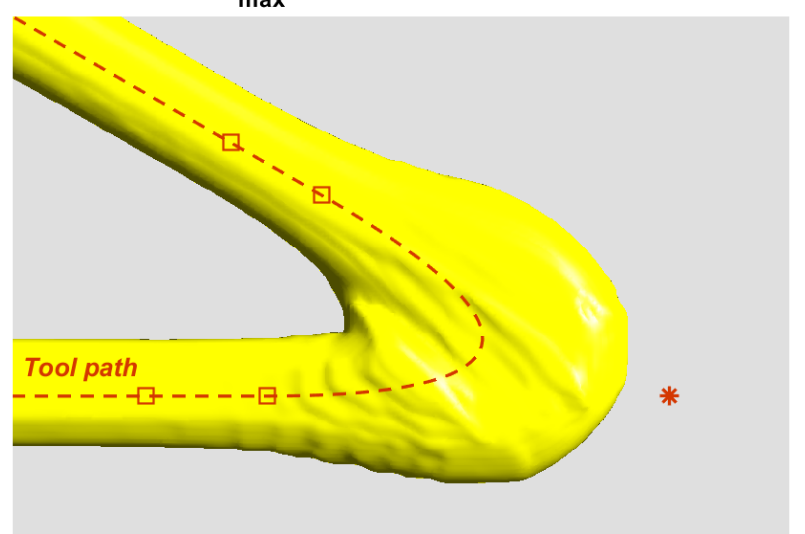

$A_{\max }=1000 \mathrm{~mm} / \mathrm{s}^{2}, \kappa=1$

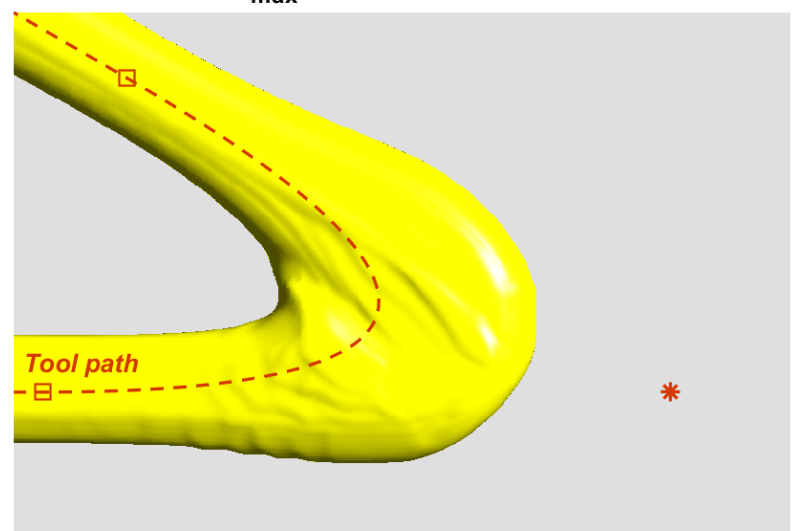

Fig. 12. Top views of $30^{\circ}$ corners, printed with different blending acceleration factors $\kappa$ and a constant extrusion rate.
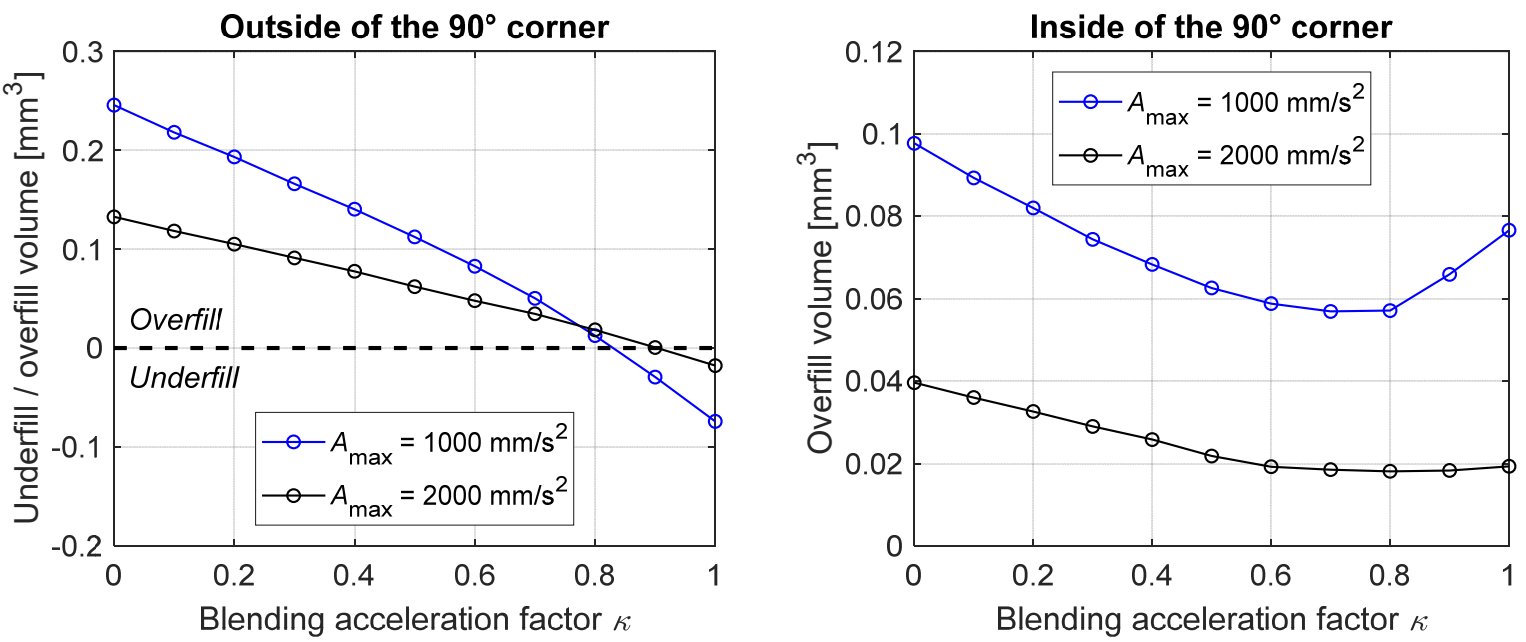

Fig. 13. Amount of underfill and overfill volume at the outside and inside of the $90^{\circ}$ corner, for a constant extrusion rate.
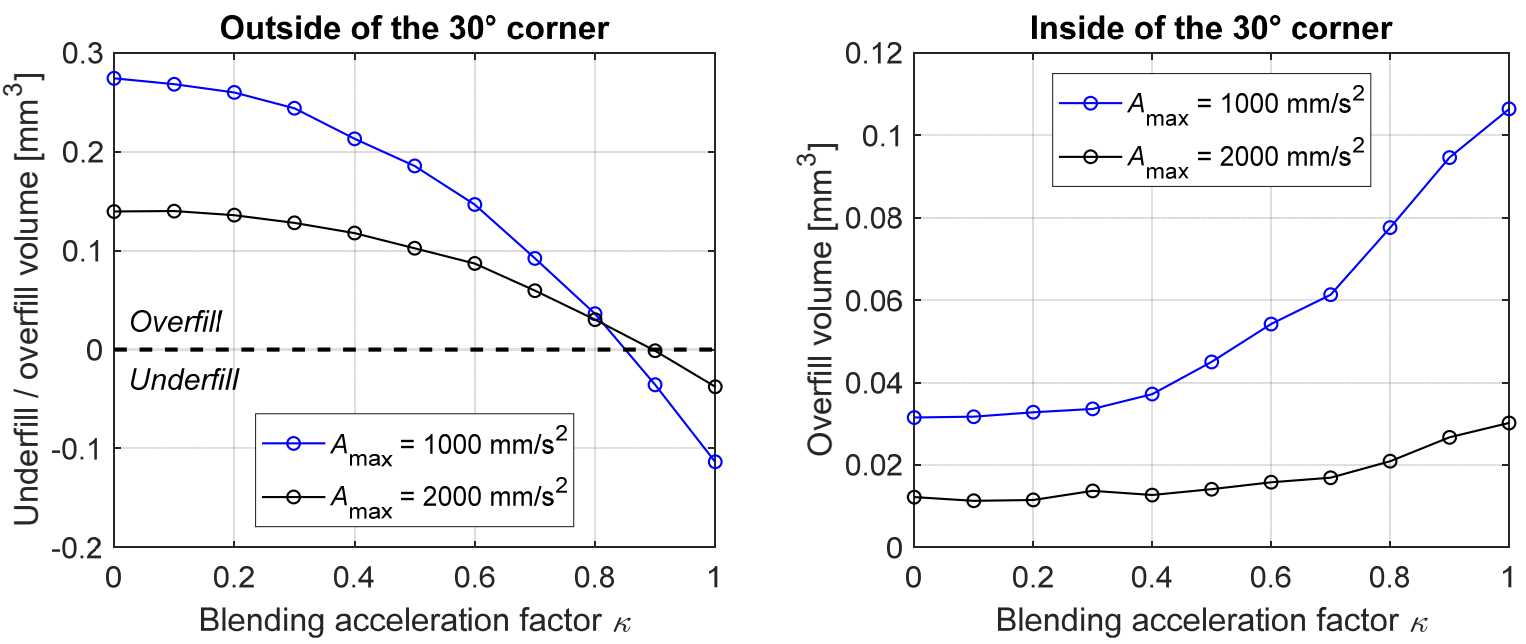

Fig. 14. Amount of underfill and overfill volume at the outside and inside of the $30^{\circ}$ corner, for a constant extrusion rate. 
Overall, the shape of the printed corners presents a compromise between moderate swelling and rounding, when the turn has a blending acceleration factor in the range $0.7<\kappa<0.9$. Moreover, the amount of underfill and overfill (as compared to the ideal turn), decreases when the maximum acceleration of the printing head is increased. Hence, when $A_{\max }$ increases, the shape of the printed corner gets closer to ideal corner obtained with the infinite acceleration. Nevertheless, a tool motion planning with overfill may be desirable, in the case where the fabricated part is post-processed by subtractive manufacturing, as suggested in [44]. In that case, a blending acceleration factor lower than 0.7 may be chosen.

\subsection{Proportional extrusion rate}

The material deposition with a proportional extrusion rate maintains a constant velocity ratio $V / U$, hence a uniform strand width along the turn, as it can be seen in Fig. 15. Thus, the corners printed with a proportional extrusion rate are not subjected to corner swelling. When $\kappa=0$ (stop-at-turn trajectory), the proportional extrusion rate yields exactly the same shape as for the ideal turn; however, it requires more printing time because of the deceleration and acceleration phases. The printed corners get rounded when $\kappa$ increases. The distance between the corner and the starting point of the curved toolpath is proportional to $\kappa^{2} V^{2} / A_{\text {max }}$; see Eq. (14). We also observe a small deviation between the toolpath and the deposition strand, which could be due to the travel time for the extruded material to reach the substrate.
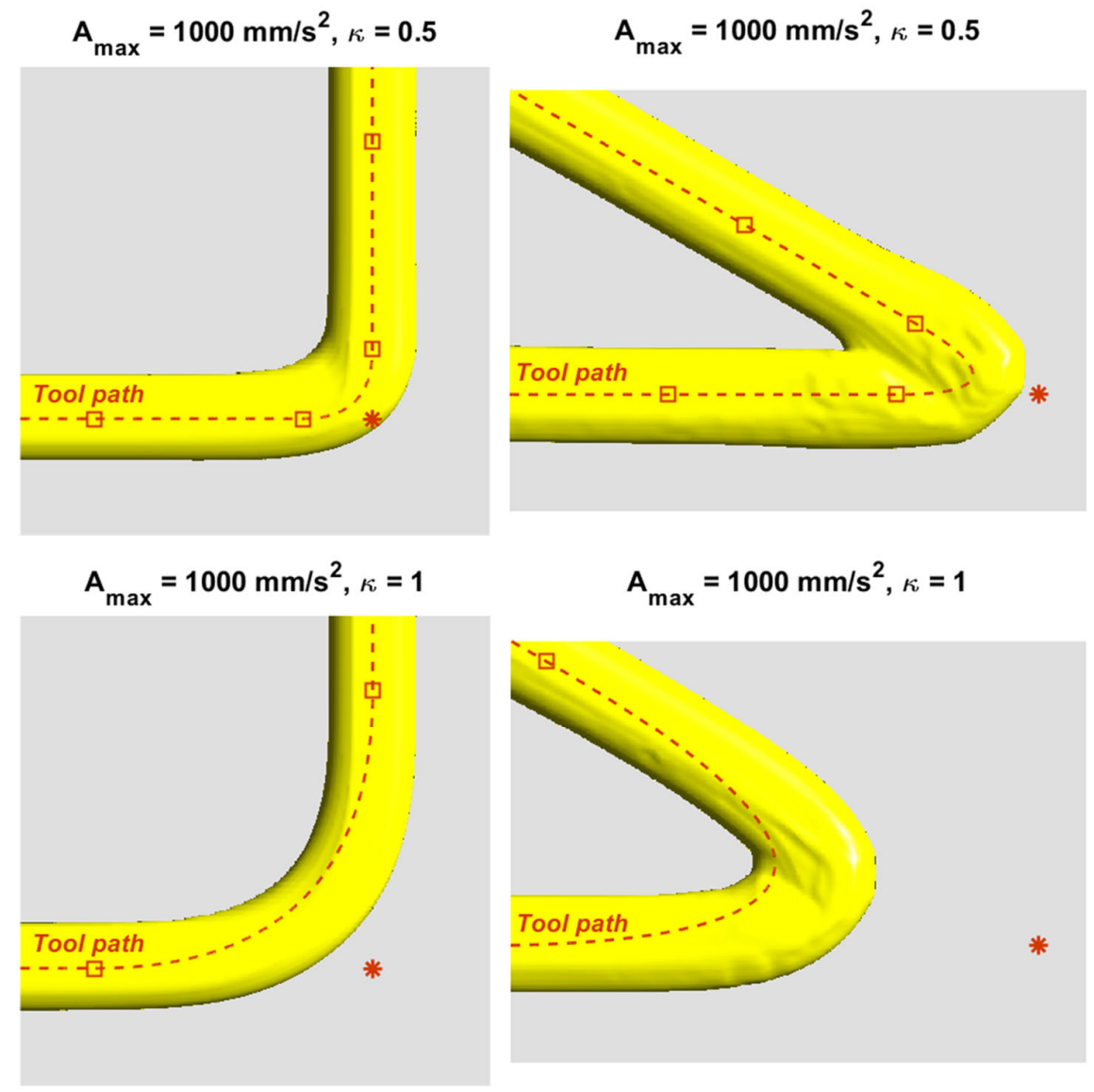

Fig. 15. Top views of $90^{\circ}$ and $30^{\circ}$ corners, printed with blending acceleration factors $\kappa=0.5$ and $\kappa=1$, and a proportional extrusion rate.

\section{Conclusions}

Overfill and underfill of material at the corners negatively impact the geometrical quality of parts fabricated by material extrusion additive manufacturing. The shape of the printed corners primarily depends on the toolpath planning around the corners, and the extrusion rate (which is not necessarily synchronized with the filament feeding rate). The maximum deviation of the smoothed toolpath (with blended accelerations) from the sharp trajectory of the corner, as well as the additional printing time required by the deceleration and acceleration phases 
in the vicinity of the corner were derived. CFD simulations have been used to isolate the effect of the toolpath smoothing and the material over-extrusion, on the corner rounding and the corner swelling.

Printing with a constant extrusion rate produces corner swelling, because the printing head motion undergoes acceleration and deceleration phases when it negotiates the turn. The amount of underfill and overfill at the inside and outside of the corner depends on the degree of toolpath smoothing, which was parametrized in our model by the blending acceleration factor. The simulations show that there is an optimal value of the blending acceleration factor, where the over-extrusion compensates for the material underfill at the outside of the corner, resulting in an improvement of the geometrical quality of the corner. The amount of underfill and overfill is reduced by increasing the maximum acceleration or decreasing the cruising speed. Nevertheless, the simulations of ideal turns (where the change of speed direction is instantaneous) show that a small amount of corner rounding is unavoidable, because of the elliptical cross-section of the deposited strand and the travelling time for the extruded material to reach the substrate.

A uniform strand width is obtained when the extrusion rate is kept proportional to the printing speed. The synchronization of the extrusion rate and the printing speed could be achieved by developing a closed-loop control system of the filament feeding mechanism. Moreover, an accurate dynamical model of the liquefier is also required to derive the suitable transfer function of the extruder. Finally, the numerical simulations predict wider and thinner deposited strand cross-sections than the idealized shapes commonly used in slicer software.

\section{Acknowledgement}

This work was supported by the Danish Council for Independent Research (DFF) | Technology and Production Sciences (FTP) (Contract No. 7017-00128). The authors gratefully acknowledge Dr. Tito Andriollo for his assistance in automating the post-processing of surface meshes with Matlab and Gmsh.

\section{Appendix A. Details in the derivation of $\Delta t_{\mathrm{CD}}$}

The derivation of $\Delta t_{\mathrm{CD}}$ in Eq. (9.2) utilizes the following sum-to-product trigonometric identities:

$$
\begin{gathered}
\cos \left(\alpha_{2}\right)-\cos \left(\alpha_{1}\right)=-2 \sin \left(\frac{\alpha_{2}+\alpha_{1}}{2}\right) \sin \left(\frac{\alpha_{2}-\alpha_{1}}{2}\right), \\
\sin \left(\alpha_{2}\right)-\sin \left(\alpha_{1}\right)=2 \cos \left(\frac{\alpha_{2}+\alpha_{1}}{2}\right) \sin \left(\frac{\alpha_{2}-\alpha_{1}}{2}\right) .
\end{gathered}
$$

Hence

$$
\max \left(\left|\cos \left(\alpha_{2}\right)-\cos \left(\alpha_{1}\right)\right|,\left|\sin \left(\alpha_{2}\right)-\sin \left(\alpha_{1}\right)\right|\right)=\frac{2\left|\sin \left(\frac{\alpha_{2}-\alpha_{1}}{2}\right)\right|}{f\left(\frac{\alpha_{2}+\alpha_{1}}{2}\right)},
$$

where $f(\alpha)$ is the function given in Eq. (10).

Moreover, it can be shown that $\left|\sin \left(\frac{\alpha_{2}-\alpha_{1}}{2}\right)\right|=\cos \left(\frac{\theta}{2}\right)$. Starting from Eq. (11):

$$
\begin{aligned}
\cos \left(\frac{\theta}{2}\right)=\cos \left(\frac{1}{2}\right. & \left.\min \left[\alpha_{2}-\alpha_{1}-\pi \bmod 2 \pi, 2 \pi-\left(\alpha_{2}-\alpha_{1}-\pi \bmod 2 \pi\right)\right]\right) \\
& =\cos \left(\min \left[\frac{\alpha_{2}-\alpha_{1}-\pi}{2} \bmod \pi, \pi-\left(\frac{\alpha_{2}-\alpha_{1}-\pi}{2} \bmod \pi\right)\right]\right) .
\end{aligned}
$$

Noticing that

$$
\cos \left(\pi-\left(\frac{\alpha_{2}-\alpha_{1}-\pi}{2} \bmod \pi\right)\right)=-\cos \left(\frac{\alpha_{2}-\alpha_{1}-\pi}{2} \bmod \pi\right)
$$

and that an angular shift of $\pm \pi$ only changes the sign of it cosine, we obtain:

$$
\left|\cos \left(\frac{\theta}{2}\right)\right|=\left|\cos \left(\frac{\alpha_{2}-\alpha_{1}-\pi}{2} \bmod \pi\right)\right|=\left|\cos \left(\frac{\alpha_{2}-\alpha_{1}-\pi}{2}\right)\right|=\left|\sin \left(\frac{\alpha_{2}-\alpha_{1}}{2}\right)\right| .
$$

Moreover, $\theta \in[0, \pi]$, thus $\cos \left(\frac{\theta}{2}\right)$ is always positive and

$$
\left|\sin \left(\frac{\alpha_{2}-\alpha_{1}}{2}\right)\right|=\cos \left(\frac{\theta}{2}\right)
$$

hence the result of $\Delta t_{\mathrm{CD}}$ in Eq. (12).

Following similar arguments, we can also show that

$$
\left|\cos \left(\frac{\alpha_{2}-\alpha_{1}}{2}\right)\right|=\sin \left(\frac{\theta}{2}\right) \text {. }
$$

\section{Appendix B. Derivation of the distances $L_{\mathrm{CD}}$ and $L_{\mathrm{CO}}$}

Since the velocity varies linearly within the curve $\mathrm{CD}$, the distance $L_{\mathrm{CD}}$ equals the product of the average velocity $\overline{V_{\mathrm{CD}}}$ along the curve CD with the travelling time $\Delta t_{\mathrm{CD}}$ between the points $\mathrm{C}$ and $\mathrm{D}$ :

$$
L_{\mathrm{CD}}=\overline{V_{\mathrm{CD}}} \Delta t_{\mathrm{CD}} \text {. }
$$


Using Eq. (5.3) and (5.4), we obtain that the average velocity along the curve CD has the following components $\overline{V_{x, \mathrm{CD}}}$

2 and $\overline{V_{y, \mathrm{CD}}}$ on the $X$ and $Y$ axes, respectively:

$$
\begin{aligned}
& \overline{V_{x, \mathrm{CD}}}=\frac{\kappa_{1} V_{x_{S 1}}+\kappa_{2} V_{x_{S 2}}}{2}, \\
& \overline{V_{y, \mathrm{CD}}}=\frac{\kappa_{1} V_{y_{S 1}}+\kappa_{2} V_{y_{S 2}}}{2} .
\end{aligned}
$$

3 Hence

$$
\overline{V_{\mathrm{CD}}}=\sqrt{{\overline{V_{x, \mathrm{CD}}}}^{2}+{\overline{V_{y, \mathrm{CD}}}}^{2}}=\sqrt{\left(\frac{\kappa_{1} V_{x_{S 1}}+\kappa_{2} V_{x_{S 2}}}{2}\right)^{2}+\left(\frac{\kappa_{1} V_{y_{S 1}}+\kappa_{2} V_{y_{S 2}}}{2}\right)^{2}} .
$$

4 Using Eq. (2.1)-(2.4) and (8.1)-(8.2), we find:

$$
\overline{V_{\mathrm{CD}}}=\frac{\kappa \min \left(V_{1}, V_{2}\right)}{2} \sqrt{\left(\cos \left(\alpha_{1}\right)+\cos \left(\alpha_{2}\right)\right)^{2}+\left(\sin \left(\alpha_{1}\right)+\sin \left(\alpha_{2}\right)\right)^{2}} .
$$

5 Moreover, the other sum-to-product trigonometric identities give:

$$
\begin{aligned}
& \cos \left(\alpha_{1}\right)+\cos \left(\alpha_{2}\right)=2 \cos \left(\frac{\alpha_{1}+\alpha_{2}}{2}\right) \cos \left(\frac{\alpha_{1}-\alpha_{2}}{2}\right), \\
& \sin \left(\alpha_{1}\right)+\sin \left(\alpha_{2}\right)=2 \sin \left(\frac{\alpha_{1}+\alpha_{2}}{2}\right) \cos \left(\frac{\alpha_{1}-\alpha_{2}}{2}\right) .
\end{aligned}
$$

Thus, using Eq. (A.8), we have:

$$
\overline{V_{\mathrm{CD}}}=\kappa \min \left(V_{1}, V_{2}\right) \sin \left(\frac{\theta}{2}\right)
$$

Hence, Eq. (13) is obtained by inserting Eq. (B.8) into Eq. (B.1). Moreover, combining Eq. (9.2) and (13) gives:

$$
L_{\mathrm{CD}}=\frac{2\left[\kappa \min \left(V_{1}, V_{2}\right)\right]^{2}}{A_{\max } f\left(\frac{\alpha_{2}+\alpha_{1}}{2}\right)} \sin \left(\frac{\theta}{2}\right) \cos \left(\frac{\theta}{2}\right)
$$

The expression of $L_{\mathrm{CO}}$ and $L_{\mathrm{OD}}$ in Eq. (14) is obtained by using the fact that the triangle COD is isosceles with the equal length $L_{\mathrm{CO}}=L_{\mathrm{OD}}$. Thus, the bisector of the angle $\widehat{\mathrm{COD}}$ is also the perpendicular bisector of the base CD of the triangle. Therefore

$$
\frac{L_{\mathrm{CD}} / 2}{L_{\mathrm{CO}}}=\sin \left(\frac{\theta}{2}\right)
$$

Finally, Eq. (14) is obtained by combining Eq. (B.9) and (B.10).

\section{Appendix C. Derivation of the maximum toolpath deviation $\varsigma$}

The coordinates $\left(x_{\mathrm{I}}, y_{\mathrm{I}}\right)$ of the middle point I of the curve CD are found by integrating the velocities given in Eq. (5.3) and (5.4) between the instant $t_{\mathrm{C}}$ and $\frac{t_{\mathrm{D}}-t_{\mathrm{C}}}{2}$ :

$$
\begin{gathered}
x_{\mathrm{I}}=x_{\mathrm{C}}+\int_{t_{\mathrm{C}}}^{\frac{t_{\mathrm{D}}-t_{\mathrm{C}}}{2}} V_{x}(t) \mathrm{d} t=x_{\mathrm{C}}+\left(t_{\mathrm{D}}-t_{\mathrm{C}}\right) \int_{0}^{\frac{1}{2}}\left[(1-\xi) \kappa_{1} V_{x_{S 1}}+\xi \kappa_{2} V_{x_{S 2}}\right] \mathrm{d} \xi \\
=x_{\mathrm{C}}+\left(t_{\mathrm{D}}-t_{\mathrm{C}}\right)\left(\frac{3}{8} \kappa_{1} V_{x_{S 1}}+\frac{1}{8} \kappa_{2} V_{x_{S 2}}\right), \\
y_{\mathrm{I}}=y_{\mathrm{C}}+\int_{t_{\mathrm{C}}}^{\frac{t_{\mathrm{D}}-t_{\mathrm{C}}}{2}} V_{y}(t) \mathrm{d} t=y_{\mathrm{C}}+\left(t_{\mathrm{D}}-t_{\mathrm{C}}\right) \int_{0}^{\frac{1}{2}}\left[(1-\xi) \kappa_{1} V_{y_{S 1}}+\xi \kappa_{2} V_{y_{S 2}}\right] \mathrm{d} \xi \\
=y_{\mathrm{C}}+\left(t_{\mathrm{D}}-t_{\mathrm{C}}\right)\left(\frac{3}{8} \kappa_{1} V_{y_{S 1}}+\frac{1}{8} \kappa_{2} V_{y_{S 2}}\right) .
\end{gathered}
$$

where $\left(x_{C}, y_{C}\right)$ are the coordinates of the point C (see Fig. 2). Using Eq. (2.1)-(2.4) and (8.1)-(8.2), we obtain:

$$
\begin{aligned}
& x_{\mathrm{I}}=x_{\mathrm{C}}+\left(t_{\mathrm{D}}-t_{\mathrm{C}}\right) \kappa \min \left(V_{1}, V_{2}\right)\left(\frac{3}{8} \cos \left(\alpha_{1}\right)+\frac{1}{8} \cos \left(\alpha_{2}\right)\right), \\
& y_{\mathrm{I}}=y_{\mathrm{C}}+\left(t_{\mathrm{D}}-t_{\mathrm{C}}\right) \kappa \min \left(V_{1}, V_{2}\right)\left(\frac{3}{8} \sin \left(\alpha_{1}\right)+\frac{1}{8} \sin \left(\alpha_{2}\right)\right) .
\end{aligned}
$$

The distance $\varsigma$ between the points 0 and I equals to:

$$
\begin{gathered}
\varsigma=\sqrt{\left(x_{\mathrm{I}}-x_{\mathrm{O}}\right)^{2}+\left(y_{\mathrm{I}}-y_{\mathrm{O}}\right)^{2}}=\sqrt{\left(x_{\mathrm{I}}-x_{\mathrm{C}}+x_{\mathrm{C}}-x_{\mathrm{O}}\right)^{2}+\left(y_{\mathrm{I}}-y_{\mathrm{C}}+y_{\mathrm{C}}-y_{\mathrm{O}}\right)^{2}} \\
=\sqrt{\left(x_{\mathrm{I}}-x_{\mathrm{C}}-L_{\mathrm{CO}} \cos \left(\alpha_{1}\right)\right)^{2}+\left(y_{\mathrm{I}}-y_{\mathrm{C}}-L_{\mathrm{CO}} \sin \left(\alpha_{1}\right)\right)^{2}} .
\end{gathered}
$$

Replacing the expression of $\Delta t_{\mathrm{CD}}=t_{\mathrm{D}}-t_{\mathrm{C}}$ and $L_{\mathrm{CO}}$ respectively given in Eq. (9.2) and (14) into Eq. (C.5) yields:

$$
\varsigma=\frac{\left[\kappa \min \left(V_{1}, V_{2}\right)\right]^{2}}{4 A_{\max } f\left(\frac{\alpha_{2}+\alpha_{1}}{2}\right)} \cos \left(\frac{\theta}{2}\right) \sqrt{\left(\cos \left(\alpha_{1}\right)-\cos \left(\alpha_{2}\right)\right)^{2}+\left(\sin \left(\alpha_{1}\right)-\sin \left(\alpha_{2}\right)\right)^{2}}
$$

Moreover, using the same sum-to-product trigonometric identities as in Eq. (A.1) and (A.2), as well as the relation of Eq. (A.7), we find 


$$
\sqrt{\left(\cos \left(\alpha_{1}\right)-\cos \left(\alpha_{2}\right)\right)^{2}+\left(\sin \left(\alpha_{1}\right)-\sin \left(\alpha_{2}\right)\right)^{2}}=2 \cos \left(\frac{\theta}{2}\right)
$$

Finally, Eq. (15) is obtained by replacing Eq. (C.7) into Eq. (C.6).

\section{Appendix D. Derivation of the additional corner travelling time $\Delta t_{\text {corner, } \kappa}$}

The additional corner travelling time is defined as $\Delta t_{\text {corner }, \kappa} \equiv \Delta t_{\kappa, \mathrm{BE}}-\Delta t_{\text {ideal,BE}}$, where $\Delta t_{\kappa, \mathrm{BE}}$ is the actual acceleration and deceleration time spend during the turn with the blending acceleration factor $\kappa$, and $\Delta t_{\text {ideal,BE }}$ is the hypothetical time that it would take for the printed head to traveled along the segments BO and OE at the full cruising speeds $V_{1}$ and $V_{2}$ (assuming an ideal turn with an instantaneous change of velocity at the corner point 0 ). The total acceleration and deceleration time of the actual turn with the blending acceleration factor $\kappa$ is:

$$
\Delta t_{\kappa, \mathrm{BE}}=\Delta t_{\mathrm{BC}}+\Delta t_{\mathrm{CD}}+\Delta t_{\mathrm{DE}}
$$

The hypothetical time spent to travel along the segments BO and OE for an ideal turn is:

$$
\Delta t_{\text {ideal, } \mathrm{BE}}=\frac{L_{\mathrm{BC}}+L_{\mathrm{CO}}}{V_{1}}+\frac{L_{\mathrm{OD}}+L_{\mathrm{DE}}}{V_{2}} .
$$

Inserting Eq. (12.1), (12.2) and (14) for $L_{\mathrm{BC}}, L_{\mathrm{DE}}, L_{\mathrm{CO}}$ and $L_{\mathrm{OD}}$ into Eq. (D.2) gives:

$$
\Delta t_{\mathrm{ideal}, \mathrm{BE}}=\frac{1+\kappa_{1}}{2} \Delta t_{\mathrm{BC}}+\frac{1+\kappa_{2}}{2} \Delta t_{\mathrm{DE}}+\left(\frac{1}{V_{1}}+\frac{1}{V_{2}}\right) \frac{\left[\kappa \min \left(V_{1}, V_{2}\right)\right]^{2}}{A_{\max } f\left(\frac{\alpha_{2}+\alpha_{1}}{2}\right)} \cos \left(\frac{\theta}{2}\right) .
$$

Combining Eq. (D.1) and (D.3) yields:

$$
\Delta t_{\text {corner }, \kappa}=\frac{1-\kappa_{1}}{2} \Delta t_{\mathrm{BC}}+\Delta t_{\mathrm{CD}}+\frac{1-\kappa_{2}}{2} \Delta t_{\mathrm{DE}}-\left(\frac{1}{V_{1}}+\frac{1}{V_{2}}\right) \frac{\left[\kappa \min \left(V_{1}, V_{2}\right)\right]^{2}}{A_{\max } f\left(\frac{\alpha_{2}+\alpha_{1}}{2}\right)} \cos \left(\frac{\theta}{2}\right) \text {. }
$$

The expressions of $\Delta t_{\mathrm{BC}}, \Delta t_{\mathrm{CD}}$ and $\Delta t_{\mathrm{DE}}$ given in Eq. (9.1)-(9.3) are inserted in Eq. (D.4):

$$
\begin{aligned}
\Delta t_{\text {corner }, \kappa}=\frac{\left(1-\kappa_{1}\right)\left[V_{1}-\kappa \min \left(V_{1}, V_{2}\right)\right]}{2 A_{\max } f\left(\alpha_{1}\right)}+\frac{2 \kappa \min \left(V_{1}, V_{2}\right)}{A_{\max } f\left(\frac{\alpha_{2}+\alpha_{1}}{2}\right)} \cos \left(\frac{\theta}{2}\right) \\
+\frac{\left(1-\kappa_{2}\right)\left[V_{2}-\kappa \min \left(V_{1}, V_{2}\right)\right]}{2 A_{\max } f\left(\alpha_{2}\right)}-\left(\frac{1}{V_{1}}+\frac{1}{V_{2}}\right) \frac{\left[\kappa \min \left(V_{1}, V_{2}\right)\right]^{2}}{A_{\max } f\left(\frac{\alpha_{2}+\alpha_{1}}{2}\right)} \cos \left(\frac{\theta}{2}\right)
\end{aligned}
$$

Furthermore, $\kappa_{1}$ and $\kappa_{2}$ can be expressed in term of $\kappa$ using Eq. (8.1) and (8.2), yielding:

$$
\begin{aligned}
& \Delta t_{\text {corner }, \kappa}=\frac{\left[V_{1}-\kappa \min \left(V_{1}, V_{2}\right)\right]^{2}}{2 V_{1} A_{\max } f\left(\alpha_{1}\right)}+\frac{\left[V_{2}-\kappa \min \left(V_{1}, V_{2}\right)\right]^{2}}{2 V_{2} A_{\max } f\left(\alpha_{2}\right)} \\
& +\frac{\kappa \min \left(V_{1}, V_{2}\right)}{A_{\max } f\left(\frac{\alpha_{2}+\alpha_{1}}{2}\right)} \cos \left(\frac{\theta}{2}\right)\left[2-\left(\frac{1}{V_{1}}+\frac{1}{V_{2}}\right) \kappa \min \left(V_{1}, V_{2}\right)\right] \text {. }
\end{aligned}
$$

The special case given in Eq. (16) is obtained by setting $V_{1}=V_{2}=V$ into Eq. (D.6).

\section{References}

[1] M.K. Agarwala, V.R. Jamalabad, N.A. Langrana, A. Safari, P.J. Whalen, S.C. Danforth, Structural quality of parts processed by fused deposition, Rapid Prototyping Journal 2 (1996) 4-19. https://doi.org/10.1108/13552549610732034.

[2] P. Kulkarni, D. Dutta, Deposition strategies and resulting part stiffnesses in fused deposition modeling, Journal of Manufacturing Science and Engineering 121 (1999) 93-103. https://doi.org/10.1115/1.2830582.

[3] B.N. Turner, S.A. Gold, A review of melt extrusion additive manufacturing processes: II. Materials, dimensional accuracy, and surface roughness, Rapid Prototyping Journal 21 (2015) 250-261. https://doi.org/10.1108/RPJ02-2013-0017.

[4] J. Go, S.N. Schiffres, A.G. Stevens, A.J. Hart, Rate limits of additive manufacturing by fused filament fabrication and guidelines for high-throughput system design, Additive Manufacturing 16 (2017) 1-11. https://doi.org/10.1016/j.addma.2017.03.007.

[5] J. Go, A.J. Hart, Fast desktop-scale extrusion additive manufacturing, Additive Manufacturing 18 (2017) 276284. https://doi.org/10.1016/j.addma.2017.10.016.

[6] B. Weiss, D.W. Storti, M.A. Ganter, Low-cost closed-loop control of a 3D printer gantry, Rapid Prototyping Journal 21 (2015) 482-490. https://doi.org/10.1108/RPJ-09-2014-0108.

[7] A. Bouhal, M.A. Jafari, W. Han, T. Fang, Tracking control and trajectory planning in layered manufacturing applications, IEEE Transactions on Industrial Electronics $46 \quad$ (1999) 445-451. https://doi.org/10.1109/41.753784.

[8] W. Han, M.A. Jafari, S.C. Danforth, A. Safari, Tool path-based deposition planning in fused deposition processes, Journal of Manufacturing Science and Engineering 124 (2002) 462-472. https://doi.org/10.1115/1.1455026.

[9] W. Han, M.A. Jafari, Coordination control of positioning and deposition in layered manufacturing, IEEE Transactions on Industrial Electronics 54 (2007) 651-659. https://doi.org/10.1109/TIE.2006.885468. 
[10] D.S. Ertay, A. Yuen, Y. Altintas, Synchronized material deposition rate control with path velocity on fused filament fabrication machines, Additive Manufacturing $19 \quad$ (2018) $205-213$. https://doi.org/10.1016/j.addma.2017.05.011.

[11] H. Giberti, L. Sbaglia, M. Parabiaghi, Trajectories generation with constant extrusion rate for experimentations on AM techniques and extrusion based technologies, Advances in Italian Mechanism Science, Springer, 2017, pp. 153-160. https://doi.org/10.1007/978-3-319-48375-7_17.

[12] H. Giberti, L. Sbaglia, M. Urgo, A path planning algorithm for industrial processes under velocity constraints with an application to additive manufacturing, Journal of Manufacturing Systems 43 (2017) 160-167. https://doi.org/10.1016/j.jmsy.2017.03.003.

[13] G.P. Greeff, M. Schilling, Closed loop control of slippage during filament transport in molten material extrusion, Additive Manufacturing 14 (2017) 31-38. https://doi.org/10.1016/j.addma.2016.12.005.

[14] K.Y. Jiang, Y.H. Gu, Controlling parameters for polymer melting and extrusion in FDM, Key Engineering Materials 259 (2004) 667-671. https://doi.org/10.4028/www.scientific.net/KEM.259-260.667.

[15] M.E. Mackay, Z.R. Swain, C.R. Banbury, D.D. Phan, D.A. Edwards, The performance of the hot end in a plasticating 3D printer, Journal of Rheology 61 (2017) 229-236. https://doi.org/10.1122/1.4973852.

[16] F. Peng, B.D. Vogt, M. Cakmak, Complex flow and temperature history during melt extrusion in material extrusion additive manufacturing, Additive Manufacturing $22 \quad$ (2018) $197-206$. https://doi.org/10.1016/j.addma.2018.05.015.

[17] D. Pollard, C. Ward, G. Herrmann, J. Etches, Filament temperature dynamics in fused deposition modelling and outlook for control, $\quad$ Procedia $\quad$ Manufacturing $11 \quad$ (2017) $536-544$. https://doi.org/10.1016/j.promfg.2017.07.147.

[18] S.R. Stewart, J.E. Wentz, J.T. Allison, Experimental and computational fluid dynamic analysis of melt flow behavior in fused deposition modelling of poly(lactic) acid, in ASME 2015 International Mechanical Engineering Congress and Exposition, American Society of Mechanical Engineers, 2015. https://doi.org/10.1115/IMECE2015-52261.

[19] T.A. Osswald, J. Puentes, J. Kattinger, Fused filament fabrication melting model, Additive Manufacturing 22 (2018) 51-59. https://doi.org/10.1016/j.addma.2018.04.030.

[20] D.D. Phan, Z.R. Swain, M.E. Mackay, Rheological and heat transfer effects in fused filament fabrication, Journal of Rheology 62 (2018) 1097-1107. https://doi.org/10.1122/1.5022982.

[21] A. Bellini, S. Guceri, M. Bertoldi, Liquefier dynamics in fused deposition, Journal of Manufacturing Science and Engineering 126 (2004) 237-246. https://doi.org/10.1115/1.1688377.

[22] J. Du, Z. Wei, X. Wang, J. Wang, Z. Chen, An improved fused deposition modeling process for forming large-size thin-walled parts, Journal of Materials Processing Technology $234 \quad$ (2016) 332-341. https://doi.org/10.1016/j.jmatprotec.2016.04.005.

[23] H. Xia, J. Lu, S. Dabiri, G. Tryggvason, Fully resolved numerical simulations of fused deposition modeling. Part I: fluid flow, Rapid Prototyping Journal 24 (2018) 463-476. https://doi.org/10.1108/RPJ-12-2016-0217.

[24] H. Xia, J. Lu, G. Tryggvason, Fully resolved numerical simulations of fused deposition modeling. Part IIsolidification, residual stresses and modeling of the nozzle, Rapid Prototyping Journal 24 (2018) $973-987$. https://doi.org/10.1108/RPJ-11-2017-0233.

[25] R. Comminal, M.P. Serdeczny, D.B. Pedersen, J. Spangenberg, Numerical modeling of the strand deposition flow in extrusion-based additive manufacturing, Additive Manufacturing $20 \quad$ (2018) 68-76. https://doi.org/10.1016/j.addma.2017.12.013.

[26] M.P. Serdeczny, R. Comminal, D.B. Pedersen, J. Spangenberg, Experimental validation of a numerical model for the strand shape in material extrusion additive manufacturing, Additive Manufacturing 24 (2018) 145-153. https://doi.org/10.1016/j.addma.2018.09.022.

[27] M.P. Serdeczny, R. Comminal, D.B. Pedersen, J. Spangenberg, Numerical simulations of the mesostructure formation in material extrusion additive manufacturing, submitted to Additive Manufacturing, 2019.

[28] R. Comminal, M.P. Serdeczny, D.B. Pedersen, J. Spangenberg, Numerical modeling of the material deposition and contouring precision in fused deposition modeling, in Proceedings of the 29th Annual International Solid Freeform Fabrication Symposium - An Additive Manufacturing Conference, 2018, pp. 1855-1864.

[29] D.-Y. Chang, B.-H. Huang, Studies on profile error and extruding aperture for the RP parts using the fused deposition modeling process, The International Journal of Advanced Manufacturing Technology 53 (2011) 1027-1037. https://doi.org/10.1007/s00170-010-2882-1.

[30] Y.-A. Jin, Y. He, J.-Z. Fu, W.-F. Gan, Z.-W. Lin, Optimization of tool-path generation for material extrusion-based additive manufacturing technology, Additive manufacturing 1-4 (2014) 32-47. https://doi.org/10.1016/j.addma.2014.08.004.

[31] D. Halvorson, A Solid Foundation for: High-Quality Corners, Printed Solid, 27 November 2016, https://www.printedsolid.com/blogs/news/a-solid-foundation-for-high-quality-corners, accessed 29 November 2018.

[32] C. Duty, C. Ajinjeru, V. Kishore, B. Compton, N. Hmeidat, X. Chen, P. Liu, A.A. Hassen, J. Lindahl, V. Kunc, What makes a material printable? A viscoelastic model for extrusion-based 3D printing of polymers, Journal of Manufacturing Processes 35 (2018) 526-537. https://doi.org/10.1016/j.jmapro.2018.08.008. 
[33] M.E. Mackay, The importance of rheological behavior in the additive manufacturing technique material extrusion, Journal of Rheology 62 (2018) 1549-1561. https://doi.org/10.1122/1.5037687.

[34] M.P. Serdeczny, R. Comminal, D.B. Pedersen, J. Spangenberg, Numerical study of the impact of shear thinning behaviour on the strand deposition flow in the extrusion-based additive manufacturing, in Euspen's 18th International Conference \& Exhibition, 2018, pp. 283-284.

[35] R. Scardovelli, S. Zaleski, Direct numerical simulation of free-surface and interfacial flow, Annual Review of Fluid Mechanics 31 (1999) 567-603. https://doi.org/10.1146/annurev.fluid.31.1.567.

[36] J.E. Pilliod Jr, E.G. Puckett, Second-order accurate volume-of-fluid algorithms for tracking material interfaces, Journal of Computational Physics 199 (2004) 465-502. https://doi.org/10.1016/j.jcp.2003.12.023.

[37] R. Comminal, J. Spangenberg, J.H. Hattel, Cellwise conservative unsplit advection for the volume of fluid method, Journal of Computational Physics 283 (2015) 582-608. https://doi.org/10.1016/j.jcp.2014.12.003.

[38] S. Muzaferija, A two-fluid Navier-Stokes solver to simulate water entry, in Proceedings of the 22nd Symposium on Naval Architecture, National Academy Press, 1999, pp. 638-651.

[39] P. Micó, stlTools, v. 1.1.0.0, MATLAB Central File Exchange, www.mathworks.com/matlabcentral/fileexchange/51200-stltools, accessed 7 September 2018.

[40] J. O'Rourke, Computational Geometry in C, Second Edition, Cambridge University Press, ISBN: 0521649765, 1998.

[41] P. Cignoni, M. Callieri, M. Corsini, M. Dellepiane, F. Ganovelli, G. Ranzuglia, MeshLab: an open-source mesh processing tool, Proceedings of the Sixth Eurographics Italian Chapter Conference, 2008, pp. 129-136. https://doi.org/10.2312/LocalChapterEvents/ItalChap/ItalianChapConf2008/129-136.

[42] C. Geuzaine, J.-F. Remacle, Gmsh: a three-dimensional finite element mesh generator with built-in pre- and postprocessing facilities, International Journal for Numerical Methods in Engineering 79 (2009) 1309-1331. https://doi.org/10.1002/nme.2579.

[43] Y.Z. Jin, J.F. Zhang, Y. Wang, Z.C. Zhu, Filament geometrical model and nozzle trajectory analysis in the fused deposition modeling process, Journal of Zhejiang University-Science A 10 (2009) 370-376. https://doi.org/10.1631/jzus.A0820346.

[44] J.-H. Kao, F.B. Prinz, Optimal motion planning for deposition in layered manufacturing, In Proceedings of the DETC'98 ASME Design Engineering Technical Conferences, 1998. 